\title{
Hepatic resection versus transarterial chemoembolization for the initial treatment of hepatocellular carcinoma: A systematic review and meta-analysis
}

\author{
Xingshun Qi ${ }^{1, *}$, Diya Wang ${ }^{2, *}$, Chunping Su${ }^{3}$, Hongyu $\mathbf{L i}^{1}$ and Xiaozhong Guo ${ }^{1}$ \\ 1 Department of Gastroenterology, General Hospital of Shenyang Military Area, Shenyang, China \\ 2 Department of Occupational and Environmental Health Sciences, Fourth Military Medical University, Xi'an, China \\ 3 Library of Fourth Military Medical University, Xi'an, China \\ * These authors have contributed equally to this work \\ Correspondence to: Xiaozhong Guo, email: guo_xiao_zhong@126.com \\ Xingshun Qi, email: xingshunqi@126.com \\ Keywords: hepatocellular carcinoma; resection; chemoembolization; BCLC stage; survival \\ Received: March 20, $2015 \quad$ Accepted: April 23, $2015 \quad$ Published: May 14, 2015 \\ This is an open-access article distributed under the terms of the Creative Commons Attribution License, which permits unrestricted use, \\ distribution, and reproduction in any medium, provided the original author and source are credited.
}

\section{ABSTRACT}

Background \& Aims: According to the Barcelona Clinic Liver Cancer (BCLC) staging system, hepatic resection and transarterial chemoembolization (TACE) should be recommended in patients with hepatocellular carcinoma (HCC) within and beyond the BCLC stage A, respectively. We conducted a systematic review and meta-analysis to compare the overall survival between HCC patients undergoing hepatic resection and TACE.

Methods: PubMed, EMBASE, and Cochrane library databases were searched. All relevant studies were considered, if they reported the survival data in HCC patients undergoing hepatic resection and TACE. Hazard ratios (HRs) with $95 \%$ confidence intervals (CIs) were calculated for the comparison of cumulative overall survival. Odds ratios (ORs) with $95 \%$ CIs were calculated for the comparison of 1-, 3-, and 5-year survival rates. Subgroup analyses were performed according to the BCLC stages and portal vein tumor thrombus (PVTT). Sensitivity analyses were performed in moderateand high-quality studies and in studies published after 2005.

Results: Fifty of 2029 retrieved papers were included. One, 15, and 34 studies were of high-, moderate-, and low-quality, respectively. The overall meta-analysis demonstrated a statistically significantly higher overall survival in hepatic resection group than in TACE group $(\mathrm{HR}=0.60,95 \% \mathrm{CI}=0.55-0.66)$. Additionally, 1-, 3-, and 5-year survival rates were statistically significantly higher in hepatic resection group than in TACE group $(O R=1.82,95 \% C I=1.56-2.14 ; O R=3.09,95 \% C I=2.60-3.67$; $\mathrm{OR}=3.48,95 \% \mathrm{CI}=2.83-4.27)$. The subgroup meta-analyses confirmed the statistical significance in $\mathrm{HCC}$ within the $\mathrm{BCLC}$ stage $A(\mathrm{HR}=0.72,95 \% \mathrm{CI}=0.64-0.80)$, in $\mathrm{HCC}$ beyond the $B C L C$ stage $A(H R=0.60,95 \% C I=0.51-0.69)$, in $\mathrm{HCC}$ within the $B C L C$ stage $B$ alone $(\mathrm{HR}=0.48,95 \% \mathrm{CI}=0.25-0.90)$, and in HCC with PVTT $(\mathrm{HR}=0.78,95 \% \mathrm{CI}=0.68-$ 0.91). The statistical significance was also confirmed by sensitivity analyses in moderate- and high-quality studies $(\mathrm{HR}=0.62,95 \% \mathrm{CI}=0.53-0.71)$ and in studies published after $2005(\mathrm{HR}=0.59,95 \% \mathrm{CI}=0.53-0.66)$.

Conclusions: Based on a systematic review and meta-analysis, hepatic resection may be considered in HCC beyond the BCLC stage A. However, given the limitations of study quality, more well-designed randomized controlled trials should be warranted to confirm these findings. 


\section{INTRODUCTION}

Nowadays, Barcelona Clinic Liver Cancer (BCLC) stage is the sole system approved by the European Association for Study of the Liver (EASL) and American Association for the Study of Liver Disease (AASLD) guidelines for the prognostic classification and treatment selection of hepatocellular carcinoma (HCC) [1-2]. According to this staging system, hepatic resection should be recommended in the BCLC stage 0 or A HCC with a single nodule (i.e., "the patients do not have liver cirrhosis or have liver cirrhosis but still have well preserved liver function, normal bilirubin and hepatic vein pressure gradient $<10 \mathrm{mmHg}$ "), and transarterial chemoembolization (TACE) should be recommended in the BCLC stage B HCC (i.e., "the patients have large/multifocal HCC but without vascular invasion or extrahepatic spread").

Recently, based on the real-world data, several large scale studies suggested that hepatic resection might be also appropriate in HCC cases beyond the BCLC stage A. First, Farinati et al. analyzed the treatment selection and prognosis of $405 \mathrm{HCC}$ cases in the BCLC stage B who were enrolled between 1986 and 2008 by the Italian Liver Cancer group [3]. Only $40 \%$ of HCC cases in the BCLC stage B underwent TACE. However, TACE achieved a significantly shorter survival time than hepatic resection in such patients (median: 27 months versus 37 months). Second, Vitale et al. analyzed the outcomes of $2090 \mathrm{HCC}$ cases in the different BCLC stages who were enrolled between 2000 and 2012 by the Italian Liver Cancer group [4]. In the BCLC stages $0, A$, and B, the net survival benefit of hepatic resection over non-surgical treatments was statistically significant. Third, Roayaie et al. analyzed the survival of 8,656 cases diagnosed with HCC between 2005 and 2011 in BRIDGE study [5]. Hepatic resection not only achieved a significantly better survival than other treatments in ideal candidates for resection, but also achieved a significantly better survival than TACE in nonideal candidates for resection.

Herein, we conducted a systematic review and metaanalysis of available literatures to clarify the survival benefits of hepatic resection over TACE in HCC patients.

\section{RESULTS}

\section{Systematic review}

Overall, 2028 papers were initially retrieved via the three major databases, including 1219 papers in PubMed, 758 in EMBASE, and 51 in Cochrane library databases. One eligible paper was also manually identified.
Among them, 50 papers were included in the systematic review (Figure 1) [6-55]. The study characteristics were summarized in Table 1. They were performed in Australia $(\mathrm{n}=1)$, Canada $(\mathrm{n}=1)$, China $(\mathrm{n}=18)$, Germany $(\mathrm{n}=7)$, India $(\mathrm{n}=1)$, Italy $(\mathrm{n}=5)$, Japan $(\mathrm{n}=6)$, Portugal $(n=1)$, South Korea $(n=8)$, Spain $(n=1)$, and USA $(n=1)$. The overall conclusions of every included study were summarized as follows: 1) the survival benefit of hepatic resection was statistically significant in 29 studies [7-8, $11,16,18-19,23-24,26,28-29,33-34,36,38,40-41$, 45-55]; 2) the survival was statistically similar between the two groups in 7 studies [12, 15, 20-22, 27, 30, 43]; and 3) the statistical difference was not reported in 14 studies [6, 9-10, 13-14, 17, 25, 31-32, 35, 37, 39, 42, 44]. The patients' characteristics and survival data of every study were shown in Supplementary Table 1. The eligibility criteria of every included study were shown in Supplementary Table 2. The criteria for treatment selection were shown in Supplementary Table 3. Only one study was of high quality, 15 studies were of moderate quality, and 34 studies were of low quality (Supplementary Table 4).

\section{Meta-analysis}

Four of 50 papers were not included in the metaanalysis, because they reported only the survival times, but not the survival rates or Kaplan-Meier curves [17, 25, 32, 37]. The remaining 46 papers were included in the metaanalysis.

\section{Overall meta-analysis}

The overall meta-analysis demonstrated a statistically significantly higher overall survival in hepatic resection group than in TACE group ( $\mathrm{HR}=0.60$, $95 \% \mathrm{CI}=0.55-0.66, \quad \mathrm{P}<0.00001) \quad$ (Figure 2). The heterogeneity was statistically significant $(\mathrm{P}<0.00001$; $\mathrm{I}^{2}=84 \%$ ). Funnel plots demonstrated that not all studies laid within 95\%CI (Supplementary Figure 1).

Additionally, the meta-analyses demonstrated that 1-, 3-, and 5-year survival rates were statistically significantly higher in hepatic resection group than in TACE group (Table 2). There were statistically significant heterogeneities in all of the 3 meta-analyses.

\section{Subgroup analysis in patients with different BCLC stages}

In $\mathrm{HCC}$ patients within the BCLC stages 0 and $\mathrm{A}$, the subgroup meta-analysis demonstrated a statistically significantly higher overall survival in hepatic resection 
Table 1: Study characteristics: An overview of included studies.

\begin{tabular}{|c|c|c|c|c|c|c|c|}
\hline $\begin{array}{l}\text { First author, } \\
\text { Journal (Year) }\end{array}$ & $\begin{array}{l}\text { Publication } \\
\text { forms }\end{array}$ & Region & Study design & Period & Follow-up time & Target population & $\begin{array}{l}\text { Survival benefits } \\
\text { (statistical } \\
\text { significance) }\end{array}$ \\
\hline $\begin{array}{l}\text { Cheng, Zhonghua } \\
\text { Zhong Liu Za Zhi } \\
\text { (2005) }\end{array}$ & Full text & China & $\begin{array}{l}\text { Retrospective } \\
\text { cohort study }\end{array}$ & $\begin{array}{l}2000.1- \\
2003.1\end{array}$ & NA & HCC with PVTT & NA. \\
\hline $\begin{array}{l}\text { Choi, World J } \\
\text { Gastroenterol (2013) }\end{array}$ & Full text & $\begin{array}{l}\text { South } \\
\text { Korea }\end{array}$ & $\begin{array}{l}\text { Retrospective } \\
\text { cohort study }\end{array}$ & $\begin{array}{l}2003.1- \\
2008.12\end{array}$ & $\begin{array}{l}\text { Median (range): } \\
38.6 \text { (1-94) months in } \\
\text { resection group }\end{array}$ & $\begin{array}{l}\text { HCC } 2-3 \text { nodules, no } \\
\text { vascular invasion, tumor } \\
\text { diameter } \leq 5 \mathrm{~cm} \text {, Child-Pugh } \\
\text { class A }\end{array}$ & $\begin{array}{l}\text { Favor hepatic } \\
\text { resection } \\
\text { (significant). }\end{array}$ \\
\hline $\begin{array}{l}\text { Ciria, J Hepatol } \\
\text { (2014) }\end{array}$ & Abstract & Spain & $\begin{array}{l}\text { Retrospective } \\
\text { cohort study }\end{array}$ & $\begin{array}{l}2006- \\
2012\end{array}$ & $\begin{array}{l}\text { Median: } \\
20.5 \text { months in all patients }\end{array}$ & BCLC stage B HCC & $\begin{array}{l}\text { Favor hepatic } \\
\text { resection } \\
\text { (significant). }\end{array}$ \\
\hline $\begin{array}{l}\text { Colella, Transpl Int } \\
\text { (1998) }\end{array}$ & Full text & Italy & $\begin{array}{l}\text { Retrospective } \\
\text { cohort study }\end{array}$ & $\begin{array}{l}1989.1- \\
1997.6\end{array}$ & $\begin{array}{l}\text { Median: } \\
43 \text { months in all patients }\end{array}$ & $\begin{array}{l}\text { HCC without extrahepatic } \\
\text { spread }\end{array}$ & NA. \\
\hline $\begin{array}{l}\text { El-Serag, J Hepatol } \\
\text { (2006) }\end{array}$ & Full text & USA & $\begin{array}{l}\text { Population-based } \\
\text { study }\end{array}$ & $\begin{array}{l}1992- \\
1999\end{array}$ & NA & $\begin{array}{l}\text { Medicare-enrolled patients } \\
\text { with } \mathrm{HCC} \text { in SEER } \\
\text { registries ( } \geq 65 \text { years old; } \\
<65 \text { years old and disable or } \\
\text { with end stage renal disease) }\end{array}$ & NA. \\
\hline $\begin{array}{l}\text { Fan, Eur J Surg } \\
\text { Oncol (2014) }\end{array}$ & Full text & $\begin{array}{l}\text { China } \\
\text { Taiwan }\end{array}$ & $\begin{array}{l}\text { Retrospective } \\
\text { cohort study }\end{array}$ & $\begin{array}{l}2007.1- \\
2012.12\end{array}$ & $\begin{array}{l}\text { Mean (range): } \\
19.5(0-67) \text { months in all } \\
\text { patients }\end{array}$ & $\begin{array}{l}\text { Aged } \geq 70 \text { years, large } \mathrm{HCCs} \\
\geq 5 \mathrm{~cm}\end{array}$ & $\begin{array}{l}\text { Favor hepatic } \\
\text { resection } \\
\text { (significant). }\end{array}$ \\
\hline $\begin{array}{l}\text { Gerunda, Liver } \\
\text { Transpl (2000) }\end{array}$ & Full text & Italy & $\begin{array}{l}\text { Prospective cohort } \\
\text { study }\end{array}$ & $\begin{array}{l}1988- \\
1997.12\end{array}$ & $\begin{array}{l}\text { Mean: } \\
4.2 \pm 1.7 \text { year in liver } \\
\text { surgery group; } \\
\text { NA in TACE group }\end{array}$ & $\begin{array}{l}\text { Child-Pugh A or B; TNM } \\
\text { stage I or II; }<3-5 \mathrm{~cm} \text { or }<3 \\
\text { nodules; no PVTT; no } \\
\text { extrahepatic diseases }\end{array}$ & Statistically similar. \\
\hline $\begin{array}{l}\text { Guglielmi, HPB } \\
\text { (2011) }\end{array}$ & Abstract & Italy & $\begin{array}{l}\text { Retrospective } \\
\text { cohort study }\end{array}$ & $\begin{array}{l}1991- \\
2009\end{array}$ & NA & HCC with cirrhosis & NA. \\
\hline $\begin{array}{l}\text { Guo, Ann Surg } \\
\text { Oncol (2014) }\end{array}$ & Full text & China & $\begin{array}{l}\text { Propensity score } \\
\text { analysis }\end{array}$ & $\begin{array}{l}2003.3- \\
2008.3\end{array}$ & $\begin{array}{l}\text { Before propensity-score } \\
\text { matching: median: } 35.0 \\
\text { months in resection group; } \\
20.8 \text { months in TACE } \\
\text { group }\end{array}$ & $\begin{array}{l}\text { BCLC stage A HCC, } \\
\text { Child-Pugh class A }\end{array}$ & NA. \\
\hline $\begin{array}{l}\text { Hasse, Langenbecks } \\
\text { Archiv für Chirurgie } \\
\text { (1996) }\end{array}$ & Full text & Germany & $\begin{array}{l}\text { Prospective cohort } \\
\text { study }\end{array}$ & $\begin{array}{l}1990.1- \\
1996.1\end{array}$ & NA & Stage pT3 or pT4 HCC & Statistically similar. \\
\hline $\begin{array}{l}\text { Helmberger, } \\
\text { Digestion (2007) }\end{array}$ & Full text & Germany & $\begin{array}{l}\text { Retrospective } \\
\text { cohort study }\end{array}$ & $\begin{array}{l}1995- \\
2006\end{array}$ & NA & HCC with VISUM stage 1 & $\begin{array}{l}\text { Favor hepatic } \\
\text { resection } \\
\text { (significant). }\end{array}$ \\
\hline Herold, Liver (2002) & Full text & Germany & $\begin{array}{l}\text { Retrospective } \\
\text { cohort study }\end{array}$ & $\begin{array}{l}1988.1- \\
1999.7\end{array}$ & $\begin{array}{l}\text { Mean (range): } \\
20(0-119) \text { months in all } \\
\text { patients }\end{array}$ & Unselected HCC & NA. \\
\hline
\end{tabular}




\begin{tabular}{|c|c|c|c|c|c|c|c|}
\hline $\begin{array}{l}\text { Ho, Ann Surg Oncol } \\
\text { (2009) }\end{array}$ & Full text & $\begin{array}{l}\text { China } \\
\text { Taiwan }\end{array}$ & $\begin{array}{l}\text { Retrospective } \\
\text { cohort study }\end{array}$ & $\begin{array}{l}1981.1- \\
2000.6\end{array}$ & $\begin{array}{l}\text { Mean (range): } \\
20.2 \text { (1-247.8) months }\end{array}$ & Multiple HCC & $\begin{array}{l}\text { Favor hepatic } \\
\text { resection } \\
\text { (significant). }\end{array}$ \\
\hline $\begin{array}{l}\text { Hsu CY, Ann Surg } \\
\text { Oncol (2012) }\end{array}$ & Full text & $\begin{array}{l}\text { China } \\
\text { Taiwan }\end{array}$ & $\begin{array}{l}\text { Propensity score } \\
\text { analysis }\end{array}$ & $\begin{array}{l}2002.1- \\
2010.10\end{array}$ & Mean: $18 \pm 16$ months & $\begin{array}{l}\text { HCC beyond the Milan } \\
\text { criteria }\end{array}$ & $\begin{array}{l}\text { Favor hepatic } \\
\text { resection } \\
\text { (significant). }\end{array}$ \\
\hline $\begin{array}{l}\text { Hsu KF, Eur J Radiol } \\
\text { (2012) }\end{array}$ & Full text & $\begin{array}{l}\text { China } \\
\text { Taiwan }\end{array}$ & $\begin{array}{l}\text { Retrospective } \\
\text { cohort study }\end{array}$ & $\begin{array}{l}2001.1- \\
2007.12\end{array}$ & $\begin{array}{l}\text { Mean: } \\
46.7 \pm 24.6 \text { months in } \\
\text { resection group; } \\
40.8 \pm 19.8 \text { months in TACE } \\
\text { group }\end{array}$ & $\begin{array}{l}\text { Resectable early-stage HCC } \\
\text { and Child-Pugh class A } \\
\text { (BCLC stage A) }\end{array}$ & Statistically similar. \\
\hline \multirow[t]{2}{*}{ Huang, EJGH (1999) } & \multirow[t]{2}{*}{ Full text } & \multirow[t]{2}{*}{$\begin{array}{l}\text { China } \\
\text { Taiwan }\end{array}$} & \multirow[t]{2}{*}{ Cohort study } & \multirow[t]{2}{*}{$\begin{array}{l}1984- \\
1993\end{array}$} & \multirow[t]{2}{*}{ NA } & $\begin{array}{l}\text { Total: Resectable HCC, } \\
\text { well-compensated liver } \\
\text { function, tumor localized to } \\
\text { a single lobe }\end{array}$ & Statistically similar. \\
\hline & & & & & & Subgroup: age $>70$ years & Statistically similar. \\
\hline \multirow{3}{*}{$\begin{array}{l}\text { Jianyong, } \\
\text { (2014) }\end{array}$} & \multirow{3}{*}{ Full text } & \multirow{3}{*}{ China } & \multirow{3}{*}{$\begin{array}{l}\text { Retrospective } \\
\text { cohort study }\end{array}$} & \multirow{3}{*}{$\begin{array}{l}2002.7- \\
2008.11\end{array}$} & \multirow{3}{*}{ NA } & $\begin{array}{l}\text { Total: BCLC stage B HCC } \\
\text { Subgroup: } 1 \text { lesion of }>5 \mathrm{~cm} \\
\text { in diameter }\end{array}$ & $\begin{array}{l}\text { Statistically similar. } \\
\text { Favor hepatic } \\
\text { resection } \\
\text { (significant). }\end{array}$ \\
\hline & & & & & & $\begin{array}{l}\text { Subgroup: } 2-3 \text { lesions (at } \\
\text { least } 1 \text { lesion was }>3 \mathrm{~cm} \text { in } \\
\text { diameter) }\end{array}$ & $\begin{array}{l}\text { Favor hepatic } \\
\text { resection } \\
\text { (significant). }\end{array}$ \\
\hline & & & & & & $\begin{array}{l}\text { Subgroup: }>3 \text { lesions of any } \\
\text { diameter }\end{array}$ & Statistically similar. \\
\hline $\begin{array}{l}\text { Jin, J Gastrointest } \\
\text { Surg (2014) }\end{array}$ & Full text & $\begin{array}{l}\text { South } \\
\text { Korea }\end{array}$ & $\begin{array}{l}\text { Retrospective } \\
\text { cohort study }\end{array}$ & $\begin{array}{l}1998.1- \\
2013.4\end{array}$ & $\begin{array}{l}\text { Mean (range): } \\
18(0.1-136) \text { months }\end{array}$ & $\begin{array}{l}\text { BCLC stage A HCC, } \\
\text { solitary, large }(>5 \mathrm{~cm})\end{array}$ & $\begin{array}{l}\text { Favor hepatic } \\
\text { resection } \\
\text { (significant). }\end{array}$ \\
\hline $\begin{array}{l}\text { Kang, Hepatol Int } \\
\text { (2010) }\end{array}$ & Abstract & $\begin{array}{l}\text { South } \\
\text { Korea }\end{array}$ & $\begin{array}{l}\text { Retrospective } \\
\text { cohort study }\end{array}$ & $\begin{array}{l}2003.1- \\
2007.12\end{array}$ & NA & Single $\mathrm{HCC}<3 \mathrm{~cm}$ & $\begin{array}{l}\text { Favor hepatic } \\
\text { resection } \\
\text { (significant). }\end{array}$ \\
\hline $\begin{array}{l}\text { Kirchner, Transplant } \\
\text { Int (2011) }\end{array}$ & Abstract & Germany & $\begin{array}{l}\text { Retrospective } \\
\text { cohort study }\end{array}$ & $\begin{array}{l}1993.3- \\
2006.11\end{array}$ & $\begin{array}{l}\text { Mean: } \\
26.7 \pm 30.7 \text { months }\end{array}$ & Unselected HCC & NA. \\
\hline $\begin{array}{l}\text { Lee JM, Hepatol Int } \\
\text { (2014) }\end{array}$ & Abstract & $\begin{array}{l}\text { South } \\
\text { Korea }\end{array}$ & Cohort study & $\begin{array}{l}2000.1- \\
2011.12\end{array}$ & NA & HCC with PVTT & $\begin{array}{l}\text { Favor hepatic } \\
\text { resection } \\
\text { (significant). }\end{array}$ \\
\hline $\begin{array}{l}\text { Lee YB, J Hepatol } \\
\text { (2014) }\end{array}$ & Abstract & $\begin{array}{l}\text { South } \\
\text { Korea }\end{array}$ & $\begin{array}{l}\text { Propensity score } \\
\text { analysis }\end{array}$ & NA & NA & $\begin{array}{l}\text { Resectable large solitary } \\
\text { HCC }\end{array}$ & Statistically similar. \\
\hline $\begin{array}{l}\text { Lin, World J Surg } \\
(2010)\end{array}$ & Full text & $\begin{array}{l}\text { China } \\
\text { Taiwan }\end{array}$ & $\begin{array}{l}\text { Retrospective } \\
\text { cohort study }\end{array}$ & $\begin{array}{l}2001.2- \\
2007.12\end{array}$ & NA & $\begin{array}{l}\text { HCC, BCLC stage B, } \\
\text { Child-Pugh A }\end{array}$ & $\begin{array}{l}\text { Favor hepatic } \\
\text { resection } \\
\text { (significant). }\end{array}$ \\
\hline $\begin{array}{l}\text { Liu, Ann Surg Oncol } \\
\text { (2014) }\end{array}$ & Full text & $\begin{array}{l}\text { China } \\
\text { Taiwan }\end{array}$ & $\begin{array}{l}\text { Propensity score } \\
\text { analysis }\end{array}$ & $\begin{array}{l}2002.2- \\
2012.12\end{array}$ & $\begin{array}{l}\text { Mean: } \\
23 \pm 22 \text { months }\end{array}$ & HCC, BCLC stage C, PVTT & $\begin{array}{l}\text { Favor hepatic } \\
\text { resection } \\
\text { (significant). }\end{array}$ \\
\hline
\end{tabular}




\begin{tabular}{|c|c|c|c|c|c|c|c|}
\hline \multirow{3}{*}{$\begin{array}{l}\text { Luo, Radiology } \\
\text { (2011) }\end{array}$} & \multirow[t]{3}{*}{ Full text } & \multirow[t]{3}{*}{ China } & \multirow{3}{*}{$\begin{array}{l}\text { Prospective cohort } \\
\text { study }\end{array}$} & \multirow{3}{*}{$\begin{array}{l}2004.1- \\
2006.12\end{array}$} & \multirow[t]{3}{*}{ NA } & \multirow{2}{*}{$\begin{array}{l}\text { Total: Large }(\geq 5 \mathrm{~cm}), \\
\text { multiple, and resectable } \\
\text { HCC } \\
\text { Subgroup: } 5-10 \mathrm{~cm}\end{array}$} & \multirow{2}{*}{$\begin{array}{l}\text { Statistically similar. } \\
\text { Statistically similar. }\end{array}$} \\
\hline & & & & & & & \\
\hline & & & & & & Subgroup: $>10 \mathrm{~cm}$ & Statistically similar. \\
\hline $\begin{array}{l}\text { Markovic, J Hepatol } \\
\text { (1998) }\end{array}$ & Full text & Italy & $\begin{array}{l}\text { Prospective cohort } \\
\text { study }\end{array}$ & $\begin{array}{l}1988.1- \\
1993.12\end{array}$ & $\begin{array}{l}\text { Mean (range): } \\
40(12-60) \text { months }\end{array}$ & $\begin{array}{l}\text { Unselected HCC (divided } \\
\text { according to the Okuda stage } \\
\text { and Child-Pugh class) }\end{array}$ & NA. \\
\hline $\begin{array}{l}\text { Martins, Liver Int } \\
\text { (2006) }\end{array}$ & Full text & Portugal & $\begin{array}{l}\text { Retrospective } \\
\text { cohort study }\end{array}$ & $\begin{array}{l}1993.1- \\
2003.12\end{array}$ & $\begin{array}{l}\text { Mean (range): } \\
66 \pm 11(22-92) \text { years }\end{array}$ & Unselected HCC & NA. \\
\hline Min, JGH (2014) & Full text & $\begin{array}{l}\text { South } \\
\text { Korea }\end{array}$ & $\begin{array}{l}\text { Propensity score } \\
\text { analysis }\end{array}$ & $\begin{array}{l}2000- \\
2009\end{array}$ & $\begin{array}{l}\text { Median (range): } \\
14.5(0-103) \text { months }\end{array}$ & $\begin{array}{l}\text { Huge } \mathrm{HCC}(\geq 10 \mathrm{~cm} \text { in } \\
\text { diameter) }\end{array}$ & $\begin{array}{l}\text { Favor hepatic } \\
\text { resection } \\
\text { (significant). }\end{array}$ \\
\hline $\begin{array}{l}\text { Nagashima, Int J } \\
\text { Oncol (1999) }\end{array}$ & Full text & Japan & $\begin{array}{l}\text { Retrospective } \\
\text { cohort study }\end{array}$ & $\begin{array}{l}1989.1- \\
1996.6\end{array}$ & NA & $\begin{array}{l}\text { Curatively unresectable } \\
\text { intrahepatic multiple HCC } \\
\text { with the main tumor } \geq 30 \\
\mathrm{~mm} \text { in size }\end{array}$ & $\begin{array}{l}\text { Favor hepatic } \\
\text { resection } \\
\text { (significant). }\end{array}$ \\
\hline $\begin{array}{l}\text { Obed, Langenbecks } \\
\text { Arch Surg (2008) }\end{array}$ & Full text & Germany & $\begin{array}{l}\text { Retrospective } \\
\text { cohort study }\end{array}$ & $\begin{array}{l}1995- \\
2000\end{array}$ & $\begin{array}{l}\text { Median (range): } \\
200 \text { (16-2054) days in } \\
\text { TACE group; } \\
399 \text { (11-2220) days in } \\
\text { resection group }\end{array}$ & $\begin{array}{l}\text { Unselected HCC (divided } \\
\text { according to UICC stage) }\end{array}$ & NA. \\
\hline $\begin{array}{l}\text { Park, J Gastroenterol } \\
\text { Hepatol (2008) }\end{array}$ & Full text & $\begin{array}{l}\text { South } \\
\text { Korea }\end{array}$ & $\begin{array}{l}\text { Prospective cohort } \\
\text { study }\end{array}$ & $\begin{array}{l}2000.11- \\
2003.12\end{array}$ & $\begin{array}{l}\text { Median: } \\
14.4 \text { months }\end{array}$ & $\begin{array}{l}\text { Subgroup: Child-Pugh class } \\
\text { A; modified UICC stage I or } \\
\text { II }\end{array}$ & $\begin{array}{l}\text { Favor hepatic } \\
\text { resection } \\
\text { (significant). } \\
\text { Favor hepatic } \\
\text { resection } \\
\text { (significant). }\end{array}$ \\
\hline $\begin{array}{l}\text { Paul, Oncology } \\
\text { (2009) }\end{array}$ & Full text & India & $\begin{array}{l}\text { Retrospective } \\
\text { cohort study } \\
(1990-2000), \\
\text { prospective cohort } \\
\text { study (2001-2005) }\end{array}$ & $\begin{array}{l}1990- \\
2005\end{array}$ & $\begin{array}{l}\text { Mean (median): } \\
7.4 \pm 10.3 \text { (3) months }\end{array}$ & Unselected HCC & NA. \\
\hline
\end{tabular}




\begin{tabular}{|c|c|c|c|c|c|c|c|}
\hline \multirow{9}{*}{ Peng, Cancer (2012) } & \multirow{9}{*}{ Full text } & \multirow{9}{*}{ China } & \multirow{9}{*}{$\begin{array}{l}\text { Retrospective } \\
\text { case-control study }\end{array}$} & \multirow{9}{*}{$\begin{array}{l}2002.12- \\
2007.12\end{array}$} & \multirow{9}{*}{$\begin{array}{l}\text { Mean (range): } \\
16.3 \pm 1.12(2.0-83.0) \\
\text { months in resection group; } \\
12.1 \pm 0.56(2.0-53.0) \\
\text { months in TACE group }\end{array}$} & $\begin{array}{l}\text { Total: Resectable HCC with } \\
\text { PVTT }\end{array}$ & $\begin{array}{l}\text { Favor hepatic } \\
\text { resection } \\
\text { (significant). }\end{array}$ \\
\hline & & & & & & $\begin{array}{l}\text { Subgroup: Resectable HCC } \\
\text { with type I PVTT }\end{array}$ & $\begin{array}{l}\text { Favor hepatic } \\
\text { resection } \\
\text { (significant). }\end{array}$ \\
\hline & & & & & & $\begin{array}{l}\text { Subgroup: Resectable HCC } \\
\text { with type II PVTT }\end{array}$ & $\begin{array}{l}\text { Favor hepatic } \\
\text { resection } \\
\text { (significant). }\end{array}$ \\
\hline & & & & & & $\begin{array}{l}\text { Subgroup: Resectable HCC } \\
\text { with type III PVTT }\end{array}$ & Statistically similar. \\
\hline & & & & & & $\begin{array}{l}\text { Subgroup: Resectable HCC } \\
\text { with type IV PVTT }\end{array}$ & Statistically similar. \\
\hline & & & & & & $\begin{array}{l}\text { Subgroup: Resectable HCC } \\
\text { with PVTT; tumor size } \leq 5 \\
\mathrm{~cm}\end{array}$ & Statistically similar. \\
\hline & & & & & & $\begin{array}{l}\text { Subgroup: Resectable HCC } \\
\text { with PVTT; tumor size }>5 \\
\mathrm{~cm}\end{array}$ & $\begin{array}{l}\text { Favor hepatic } \\
\text { resection } \\
\text { (significant). }\end{array}$ \\
\hline & & & & & & $\begin{array}{l}\text { Subgroup: Resectable HCC } \\
\text { with PVTT; single tumor }\end{array}$ & $\begin{array}{l}\text { Favor hepatic } \\
\text { resection } \\
\text { (significant). }\end{array}$ \\
\hline & & & & & & $\begin{array}{l}\text { Subgroup: Resectable HCC } \\
\text { with PVTT; multiple tumor }\end{array}$ & Statistically similar. \\
\hline $\begin{array}{l}\text { Perry, Liver Int } \\
\text { (2007) }\end{array}$ & Full text & Australia & $\begin{array}{l}\text { Prospective cohort } \\
\text { study }\end{array}$ & $\begin{array}{l}\text { Since } \\
1998\end{array}$ & $\begin{array}{l}\text { Median: } \\
33 \text { months for survivors; } \\
9 \text { months for patients who } \\
\text { died }\end{array}$ & Unselected HCC & NA. \\
\hline $\begin{array}{l}\text { Sako, Anticancer } \\
\text { Research (2003) }\end{array}$ & Full text & Japan & $\begin{array}{l}\text { Retrospective } \\
\text { cohort study }\end{array}$ & $\begin{array}{l}1993.4- \\
2001.10\end{array}$ & $\begin{array}{l}\text { Mean (range): } \\
4.2(0.3-10.8) \text { years in all } \\
\text { patients }\end{array}$ & $\begin{array}{l}\text { HCV-related, single, small } \\
\text { HCC }\end{array}$ & $\begin{array}{l}\text { Favor hepatic } \\
\text { resection } \\
\text { (significant). }\end{array}$ \\
\hline \multirow{4}{*}{$\begin{array}{l}\text { Sasaki, J } \\
\text { Hepatobiliary } \\
\text { Pancreat Surg (1998) }\end{array}$} & \multirow{4}{*}{ Full text } & \multirow{4}{*}{ Japan } & \multirow{4}{*}{$\begin{array}{l}\text { Retrospective } \\
\text { cohort study }\end{array}$} & \multirow{4}{*}{$\begin{array}{l}1980.1- \\
1994.4\end{array}$} & \multirow{4}{*}{ NA } & Total: unselected HCC & $\begin{array}{l}\text { Favor hepatic } \\
\text { resection } \\
\text { (significant). }\end{array}$ \\
\hline & & & & & & $\begin{array}{l}\text { Subgroup: Liver Cancer } \\
\text { Study Group of Japan stage I } \\
\text { HCC }\end{array}$ & Statistically similar. \\
\hline & & & & & & $\begin{array}{l}\text { Subgroup: Liver Cancer } \\
\text { Study Group of Japan stage } \\
\text { II HCC }\end{array}$ & $\begin{array}{l}\text { Favor hepatic } \\
\text { resection } \\
\text { (significant). }\end{array}$ \\
\hline & & & & & & $\begin{array}{l}\text { Subgroup: Liver Cancer } \\
\text { Study Group of Japan stage } \\
\text { III HCC }\end{array}$ & $\begin{array}{l}\text { Favor hepatic } \\
\text { resection } \\
\text { (significant). }\end{array}$ \\
\hline
\end{tabular}




\begin{tabular}{|c|c|c|c|c|c|c|c|}
\hline $\begin{array}{l}\text { Schumacher, Ann } \\
\text { Hepatol (2010) }\end{array}$ & Full text & Canada & $\begin{array}{l}\text { Retrospective } \\
\text { cohort study }\end{array}$ & $\begin{array}{l}1996.1- \\
2006.12\end{array}$ & NA & Unselected HCC & NA. \\
\hline $\begin{array}{l}\text { Sotiropoulos, Dig } \\
\text { Dis Sci (2009) }\end{array}$ & Full text & Germany & $\begin{array}{l}\text { Retrospective } \\
\text { cohort study }\end{array}$ & $\begin{array}{l}1998.4- \\
2007.5\end{array}$ & $\begin{array}{l}\text { Median (range): } \\
15.3(0.2-144) \text { months }\end{array}$ & $\begin{array}{l}\text { HCC with cirrhosis and no } \\
\text { prior tumor treatments }\end{array}$ & Statistically similar. \\
\hline $\begin{array}{l}\text { Toro, BMC Surg } \\
\text { (2014) }\end{array}$ & Full text & Italy & $\begin{array}{l}\text { Retrospective } \\
\text { cohort study }\end{array}$ & $\begin{array}{l}2002.1- \\
2012.12\end{array}$ & NA & $\begin{array}{l}\text { HCC aged }>18 \text { years, } \\
\text { Child-Pugh class A or B }\end{array}$ & NA. \\
\hline \multirow{4}{*}{$\begin{array}{l}\text { Ueno, J } \\
\text { Hepatobiliary } \\
\text { Pancreat Surg (2002) }\end{array}$} & \multirow{4}{*}{ Full text } & \multirow{4}{*}{ Japan } & \multirow{4}{*}{$\begin{array}{l}\text { Prospective cohort } \\
\text { study }\end{array}$} & \multirow{4}{*}{$\begin{array}{l}1990.1- \\
1998.10\end{array}$} & \multirow{4}{*}{ NA } & $\begin{array}{l}\text { Total: HCC; Child class B } \\
\text { and C cirrhosis without } \\
\text { lymph node or distant } \\
\text { metastasis }\end{array}$ & $\begin{array}{l}\text { Favor hepatic } \\
\text { resection } \\
\text { (significant). }\end{array}$ \\
\hline & & & & & & $\begin{array}{l}\text { Subgroup: Prognostic score } \\
=0\end{array}$ & $\begin{array}{l}\text { Favor hepatic } \\
\text { resection } \\
\text { (significant). }\end{array}$ \\
\hline & & & & & & $\begin{array}{l}\text { Subgroup: Prognostic score } \\
=1-2\end{array}$ & $\begin{array}{l}\text { Favor hepatic } \\
\text { resection } \\
\text { (significant). }\end{array}$ \\
\hline & & & & & & $\begin{array}{l}\text { Subgroup: Prognostic score } \\
=3\end{array}$ & Statistically similar. \\
\hline $\begin{array}{l}\text { Utsunomiya, Ann } \\
\text { Surg (2014) }\end{array}$ & Full text & Japan & $\begin{array}{l}\text { Prospective cohort } \\
\text { study }\end{array}$ & $\begin{array}{l}2000.1- \\
2005.12\end{array}$ & $\begin{array}{l}\text { Mean: } \\
1.9 \pm 1.6 \text { years in resection } \\
\text { group; } \\
1.5 \pm 1.4 \text { years in TACE } \\
\text { group }\end{array}$ & $\begin{array}{l}\text { Non-HBV and non-HCV } \\
\text { HCC }\end{array}$ & $\begin{array}{l}\text { Favor hepatic } \\
\text { resection } \\
\text { (significant). }\end{array}$ \\
\hline $\begin{array}{l}\text { Wang, Academic } \\
\text { Journal of Second } \\
\text { Military Medical } \\
\text { University (2012) }\end{array}$ & Full text & China & $\begin{array}{l}\text { Propensity score } \\
\text { analysis }\end{array}$ & $\begin{array}{l}2003- \\
2011\end{array}$ & NA & Early-stage HCC & $\begin{array}{l}\text { Favor hepatic } \\
\text { resection } \\
\text { (significant). }\end{array}$ \\
\hline $\begin{array}{l}\text { Wang, Dig Liver Dis } \\
\text { (2013) }\end{array}$ & Full text & $\begin{array}{l}\text { China } \\
\text { Taiwan }\end{array}$ & $\begin{array}{l}\text { Retrospective } \\
\text { cohort study }\end{array}$ & $\begin{array}{l}2003- \\
2008\end{array}$ & NA & $\begin{array}{l}\text { BCLC stage C; naïve HCC; } \\
\text { ECOG score } \leq 2 \text { and } \\
\text { Child-Pugh class A }\end{array}$ & $\begin{array}{l}\text { Favor hepatic } \\
\text { resection } \\
\text { (significant). }\end{array}$ \\
\hline $\begin{array}{l}\text { Worns, Scand J } \\
\text { Gastroenterol (2012) }\end{array}$ & Full text & Germany & $\begin{array}{l}\text { Retrospective } \\
\text { cohort study }\end{array}$ & $\begin{array}{l}1997.1- \\
2009.12\end{array}$ & NA & $\mathrm{HCC}$ in non-cirrhotic liver & $\begin{array}{l}\text { Favor hepatic } \\
\text { resection } \\
\text { (significant). }\end{array}$ \\
\hline $\begin{array}{l}\text { Yamagiwa, J } \\
\text { Gastroenterol } \\
\text { Hepatol (2008) }\end{array}$ & Full text & Japan & $\begin{array}{l}\text { Retrospective } \\
\text { cohort study }\end{array}$ & $\begin{array}{l}1995.1- \\
2004.12\end{array}$ & $\begin{array}{l}\text { Median: } \\
1008 \text { days in resection } \\
\text { group; } \\
609 \text { days in TACE group }\end{array}$ & Unselected HCC & $\begin{array}{l}\text { Favor hepatic } \\
\text { resection } \\
\text { (significant). }\end{array}$ \\
\hline $\begin{array}{l}\text { Yang, Radiology } \\
\text { (2014) }\end{array}$ & Full text & $\begin{array}{l}\text { South } \\
\text { Korea }\end{array}$ & $\begin{array}{l}\text { Retrospective } \\
\text { cohort study* }\end{array}$ & $\begin{array}{l}2005.1- \\
2006.12\end{array}$ & NA & $\begin{array}{l}\mathrm{HCC} \leq 3 \mathrm{~cm} \text { in diameter, no } \\
\text { vascular invasion, single } \\
\text { nodule }\end{array}$ & $\begin{array}{l}\text { Favor hepatic } \\
\text { resection } \\
\text { (significant). }\end{array}$ \\
\hline $\begin{array}{l}\text { Ye, World J } \\
\text { Gastroenterol (2014) }\end{array}$ & Full text & China & $\begin{array}{l}\text { Retrospective } \\
\text { cohort study }\end{array}$ & $\begin{array}{l}2007- \\
2009\end{array}$ & NA & HCC with PVTT & $\begin{array}{l}\text { Favor hepatic } \\
\text { resection } \\
\text { (significant). }\end{array}$ \\
\hline
\end{tabular}




\begin{tabular}{|c|c|c|c|c|c|c|c|}
\hline Yin, J Hepatol (2014) & Full text & China & $\begin{array}{l}\text { Randomized } \\
\text { controlled trial }\end{array}$ & $\begin{array}{l}2008.11- \\
2010.9\end{array}$ & $\begin{array}{l}\text { Median }(95 \% \mathrm{CI}) \text { : } \\
33.3(28.1-53.8) \text { months in } \\
\text { surgery group; } \\
13.5 \text { (9.5-18.4) months in } \\
\text { TACE group }\end{array}$ & $\begin{array}{l}\text { Resectable multiple HCC } \\
\text { outside of Milan criteria }\end{array}$ & $\begin{array}{l}\text { Favor hepatic } \\
\text { resection } \\
\text { (significant). }\end{array}$ \\
\hline \multirow{4}{*}{$\begin{array}{l}\text { Zhang, J Surg Res } \\
\text { (2014) }\end{array}$} & \multirow{4}{*}{ Full text } & \multirow{4}{*}{ China } & \multirow{4}{*}{$\begin{array}{l}\text { Retrospective } \\
\text { cohort study }\end{array}$} & \multirow{4}{*}{$\begin{array}{l}2005.1- \\
2010.3\end{array}$} & \multirow{4}{*}{$\begin{array}{l}\text { Median (range): } \\
28(3-84) \text { months in all } \\
\text { patients }\end{array}$} & $\begin{array}{l}\text { Total: HCC; multiple tumors } \\
\text { involving both lobes of the } \\
\text { liver }\end{array}$ & $\begin{array}{l}\text { Favor hepatic } \\
\text { resection } \\
\text { (significant). }\end{array}$ \\
\hline & & & & & & Subgroup: BCLC stage A & $\begin{array}{l}\text { Favor hepatic } \\
\text { resection } \\
\text { (significant). }\end{array}$ \\
\hline & & & & & & Subgroup: BCLC stage B & $\begin{array}{l}\text { Favor hepatic } \\
\text { resection } \\
\text { (significant). }\end{array}$ \\
\hline & & & & & & Subgroup: BCLC stage C & $\begin{array}{l}\text { Favor hepatic } \\
\text { resection } \\
\text { (significant). }\end{array}$ \\
\hline $\begin{array}{l}\text { Zhong, Ann Surg } \\
\text { (2014) }\end{array}$ & Full text & China & $\begin{array}{l}\text { Propensity score } \\
\text { analysis }\end{array}$ & $\begin{array}{l}2000.1- \\
2007.12\end{array}$ & $\begin{array}{l}\text { Median (range): } \\
31.2(1-120.3) \text { months in } \\
\text { the overall analysis }{ }^{\#}\end{array}$ & BCLC stage $\mathrm{B} / \mathrm{C}$ HCC & $\begin{array}{l}\text { Favor hepatic } \\
\text { resection } \\
\text { (significant). }\end{array}$ \\
\hline
\end{tabular}

Abbreviations: BCLC, Barcelona Clinic Liver Cancer; HCC, hepatocellular carcinoma; NA, not available; PVTT, portal vein tumor thrombus; UICC, Union International Centre Cancer; VISUM, Vienna survival model.

Notes: *, data in propensity score analysis was not collected due to the absence of relevant data.

\# the follow-up length was not available in the propensity score analysis.

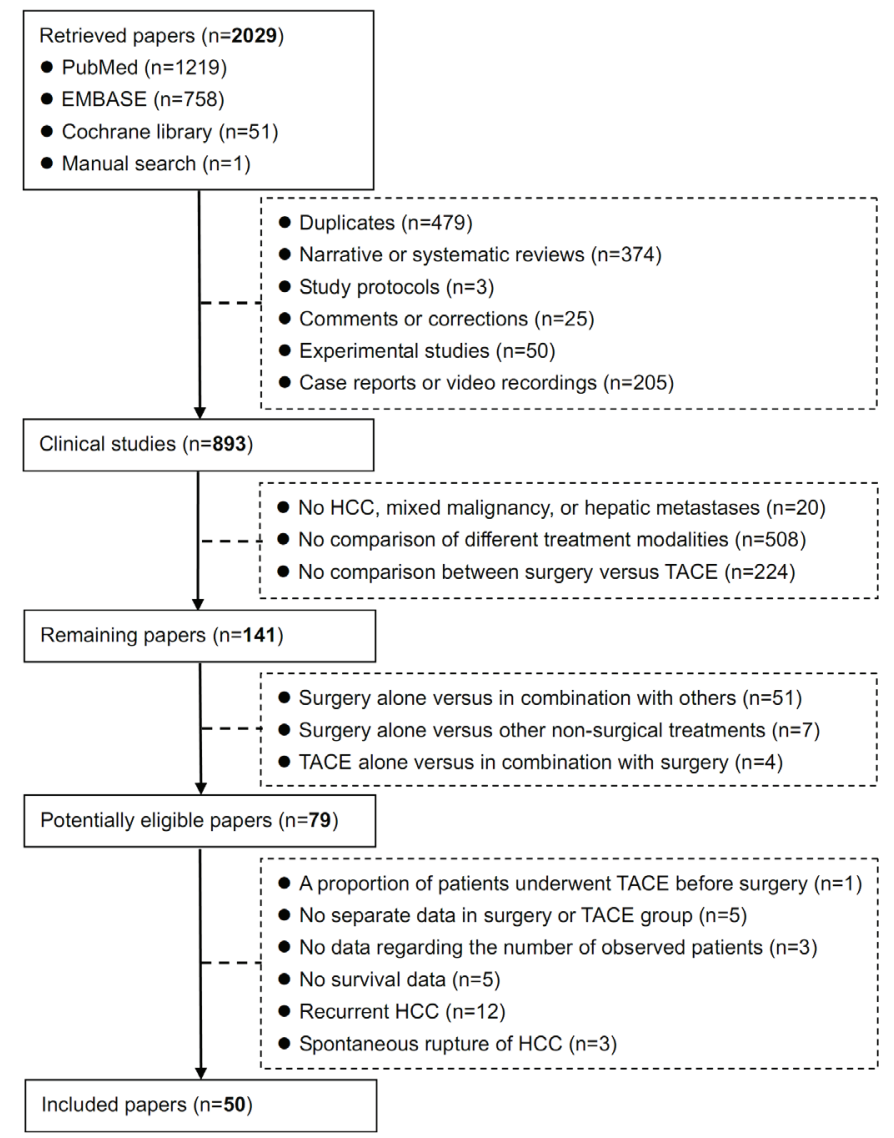

\section{Figure 1: Flowchart of study inclusion.}


group than in TACE group $(\mathrm{HR}=0.72,95 \% \mathrm{CI}=0.64$ $0.80, \mathrm{P}<0.00001$ ) (Figure 3). The heterogeneity was not statistically significant $\left(\mathrm{P}=0.92 ; \mathrm{I}^{2}=0 \%\right)$. Funnel plots demonstrated that all studies laid within 95\%CI (Supplementary Figure 2).
Additionally, the meta-analyses demonstrated that 1-, 3-, and 5-year survival rates were statistically significantly higher in hepatic resection group than in TACE group (Table 2). There was a statistically significant heterogeneity in the meta-analysis of 5-year survival rate,

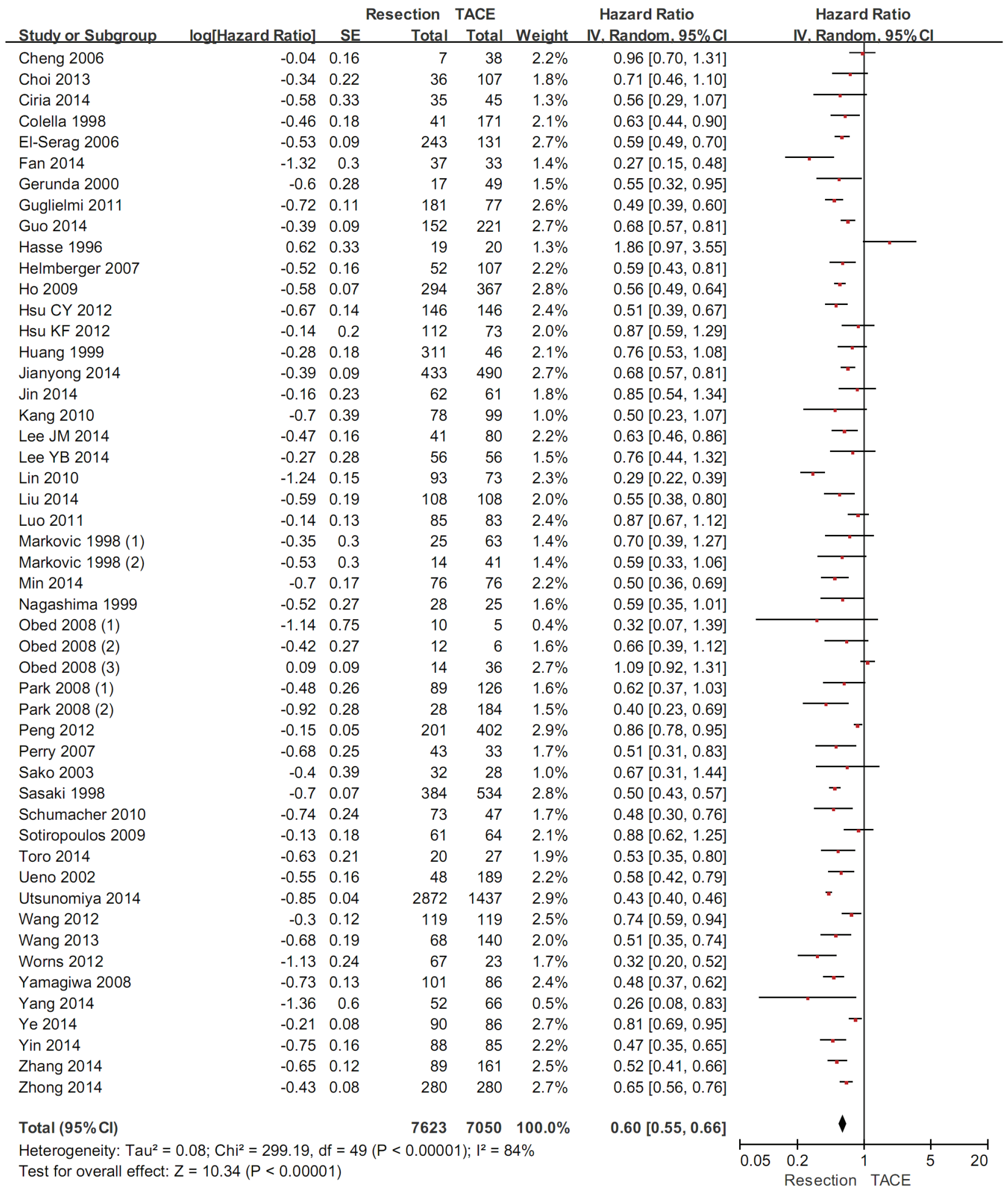

Figure 2: The overall meta-analysis comparing the overall survival between HCC patients undergoing hepatic resection and TACE. 
but not in those of 1- and 3-year survival rates.

In $\mathrm{HCC}$ patients beyond the BCLC stage A, the subgroup meta-analysis demonstrated a statistically significantly higher overall survival in hepatic resection group than in TACE group $(\mathrm{HR}=0.60,95 \% \mathrm{CI}=0.51$ $0.69, \mathrm{P}<0.00001$ ) (Figure 3). The heterogeneity was statistically significant $\left(\mathrm{P}<0.00001 ; \mathrm{I}^{2}=83 \%\right)$. Funnel plots demonstrated that not all studies laid within $95 \% \mathrm{CI}$ (Supplementary Figure 3).

Additionally, the meta-analyses demonstrated that 1-, 3-, and 5-year survival rates were statistically significantly higher in hepatic resection group than in TACE group (Table 2). There were statistically significant heterogeneities in all of the three meta-analyses.

There was a statistically significant subgroup difference $\left(\mathrm{P}<0.00001 ; \mathrm{I}^{2}=75 \%\right)$.

\section{Subgroup analysis in patients with BCLC stage $B$ alone}

In HCC patients with BCLC stage B alone, the subgroup meta-analysis demonstrated a statistically significantly higher overall survival in hepatic resection group than in TACE group $(\mathrm{HR}=0.48,95 \% \mathrm{CI}=0.25$ $0.90, \quad \mathrm{P}=0.02$ ) (Figure 4). The heterogeneity was statistically significant $\left(\mathrm{P}<0.00001 ; \mathrm{I}^{2}=92 \%\right)$. Funnel plots demonstrated that not all studies laid within $95 \% \mathrm{CI}$ (Supplementary Figure 4).

Additionally, the meta-analyses demonstrated that

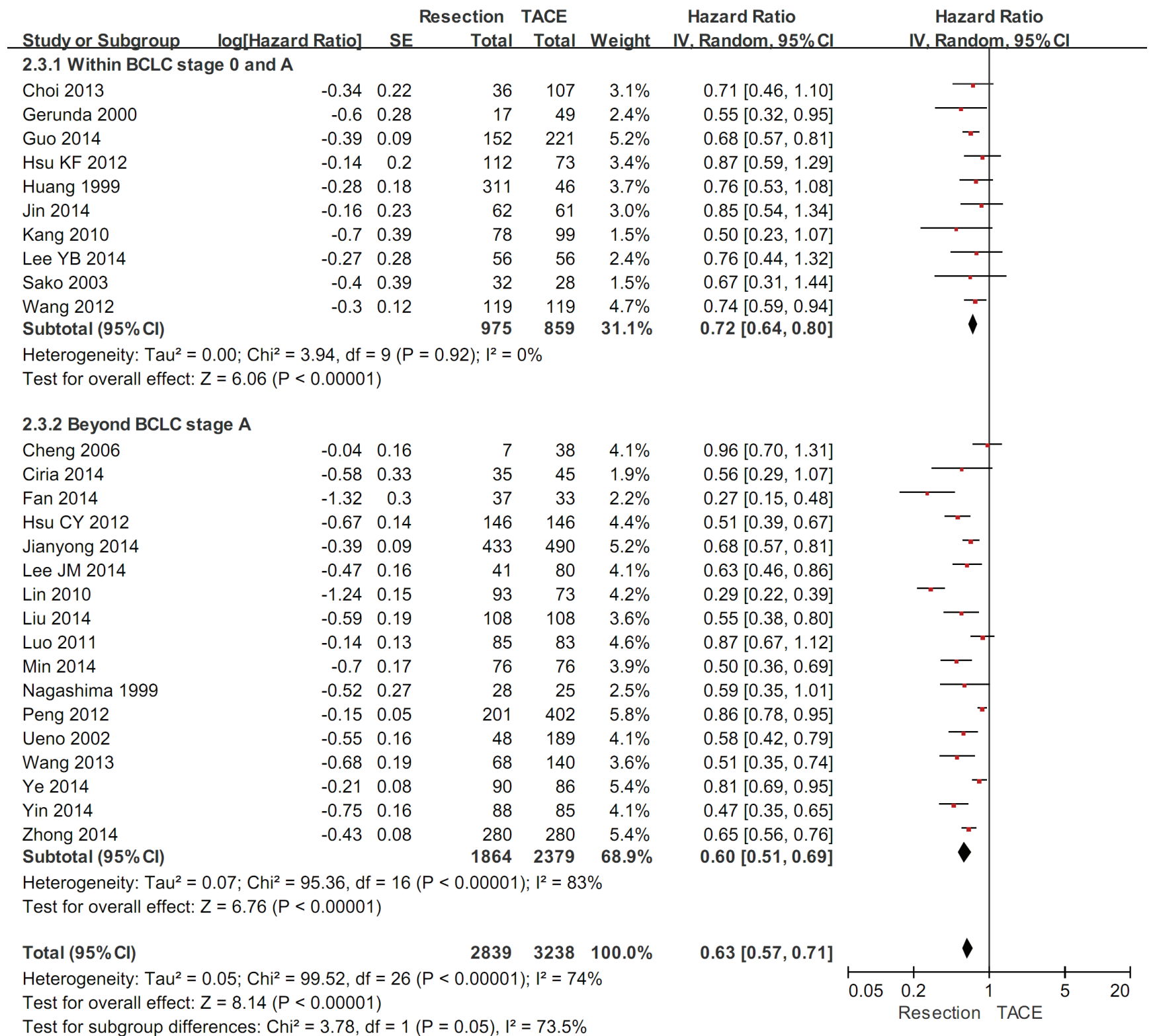

Figure 3: The subgroup meta-analysis comparing the overall survival between HCC patients within and beyond the BCLC stage A undergoing hepatic resection and TACE. 
Table 2: Comparisons of 1-, 3,- and 5-year survival between hepatic resection and TACE groups: Results of meta-analyses.

\begin{tabular}{|c|c|c|c|c|c|c|c|c|c|}
\hline \multirow{2}{*}{ Variables } & \multirow{2}{*}{$\begin{array}{c}\text { No. } \\
\text { included } \\
\text { studies }\end{array}$} & \multicolumn{2}{|c|}{$\begin{array}{c}\text { Hepatic resection } \\
\text { group }\end{array}$} & \multicolumn{2}{|c|}{ TACE group } & \multicolumn{2}{|c|}{ Effect size } & \multicolumn{2}{|c|}{ Heterogeneity } \\
\hline & & $\begin{array}{l}\text { No. Pts. } \\
\text { observed }\end{array}$ & $\begin{array}{l}\text { No. Pts } \\
\text { survival }\end{array}$ & $\begin{array}{l}\text { No. Pts. } \\
\text { observed }\end{array}$ & $\begin{array}{l}\text { No. Pts } \\
\text { survival }\end{array}$ & $\begin{array}{l}\text { Odds ratio } \\
(95 \% \mathrm{CI})\end{array}$ & P value & $I^{2}$ & P value \\
\hline \multicolumn{10}{|l|}{ All patients } \\
\hline 1-year survival & 41 & 6879 & 5689 & 6149 & 4279 & $1.82(1.56-2.14)$ & $<0.00001$ & $50 \%$ & 0.0001 \\
\hline 3-year survival & 41 & 7392 & 4778 & 6719 & 2542 & $3.09(2.60-3.67)$ & $<0.00001$ & $70 \%$ & $<0.00001$ \\
\hline 5-year survival & 32 & 6551 & 3562 & 5710 & 1359 & $3.48(2.83-4.27)$ & $<0.00001$ & $68 \%$ & $<0.00001$ \\
\hline \multicolumn{10}{|c|}{ Within BCLC stage $A$} \\
\hline 1-year survival & 10 & 975 & 815 & 859 & 681 & $1.38(1.00-1.91)$ & 0.05 & $16 \%$ & 0.3 \\
\hline 3-year survival & 10 & 975 & 586 & 859 & 398 & $1.92(1.44-2.57)$ & $<0.0001$ & $36 \%$ & 0.12 \\
\hline 5-year survival & 9 & 856 & 434 & 740 & 232 & $2.55(1.61-4.06)$ & $<0.0001$ & $68 \%$ & 0.002 \\
\hline \multicolumn{10}{|c|}{ Beyond BCLC stage $A$} \\
\hline 1-year survival & 16 & 1827 & 1354 & 2346 & 1422 & $2.06(1.57-2.71)$ & $<0.00001$ & $64 \%$ & 0.0002 \\
\hline 3-year survival & 15 & 1789 & 940 & 2201 & 659 & $3.51(2.45-5.02)$ & $<0.00001$ & $77 \%$ & $<0.00001$ \\
\hline 5-year survival & 8 & 1329 & 565 & 1723 & 379 & $2.89(2.02-4.13)$ & $<0.00001$ & $66 \%$ & 0.004 \\
\hline \multicolumn{10}{|c|}{ BCLC stage $B$ alone } \\
\hline 1-year survival & 3 & 561 & 474 & 608 & 472 & $2.38(0.64-8.86)$ & 0.2 & $91 \%$ & $<0.0001$ \\
\hline 3-year survival & 3 & 561 & 378 & 608 & 326 & $4.66(1.01-21.5)$ & 0.05 & $88 \%$ & 0.0002 \\
\hline 5 -year survival & 1 & 433 & 265 & 490 & 221 & $1.92(1.48-2.50)$ & $<0.00001$ & NA & NA \\
\hline \multicolumn{10}{|l|}{ PVTT } \\
\hline 1-year survival & 5 & 447 & 227 & 714 & 277 & $1.73(1.17-2.57)$ & 0.006 & $37 \%$ & 0.17 \\
\hline 3-year survival & 4 & 440 & 129 & 676 & 90 & $2.72(1.59-4.66)$ & 0.0003 & $41 \%$ & 0.17 \\
\hline 5-year survival & 2 & 309 & 86 & 510 & 40 & $7.34(0.79-68.16)$ & 0.08 & $88 \%$ & 0.004 \\
\hline \multicolumn{10}{|c|}{ Moderate-and high-quality studies } \\
\hline 1-year survival & 15 & 2097 & 1612 & 2369 & 1537 & $1.95(1.50-2.52)$ & $<0.00001$ & $64 \%$ & 0.0004 \\
\hline 3-year survival & 16 & 2134 & 1152 & 2402 & 792 & $3.04(2.18-4.23)$ & $<0.00001$ & $78 \%$ & $<0.00001$ \\
\hline 5-year survival & 10 & 1631 & 732 & 1930 & 479 & $2.82(1.99-4.00)$ & $<0.00001$ & $72 \%$ & 0.0002 \\
\hline \multicolumn{10}{|c|}{ Studies published after 2005} \\
\hline 1-year survival & 34 & 6385 & 5296 & 5688 & 3923 & $1.90(1.61-2.23)$ & $<0.00001$ & $51 \%$ & 0.0002 \\
\hline 3-year survival & 33 & 6492 & 4191 & 5573 & 2150 & $3.11(2.58-3.74)$ & $<0.00001$ & $68 \%$ & $<0.00001$ \\
\hline 5-year survival & 25 & 5690 & 3166 & 4668 & 1161 & $3.62(2.85-4.61)$ & $<0.00001$ & $71 \%$ & $<0.00001$ \\
\hline
\end{tabular}

\begin{tabular}{|c|c|c|c|c|c|c|c|c|c|c|}
\hline Studv or Subgroup & loa[Hazard Ratiol & SE & $\begin{array}{l}\text { ction } \\
\text { Total }\end{array}$ & TACE & Weiaht & $\begin{array}{l}\text { Hazard Ratio } \\
\text { IV }\end{array}$ & & Hazard & rd Ratio & \\
\hline Ciria 2014 & -0.58 & 0.33 & 35 & 45 & $27.4 \%$ & $0.56[0.29,1.07]$ & & & & \\
\hline Jianyong 2014 & -0.39 & 0.09 & 433 & 490 & $37.2 \%$ & $0.68[0.57,0.81]$ & & & & \\
\hline Lin 2010 & -1.24 & 0.15 & 93 & 73 & $35.4 \%$ & $0.29[0.22,0.39]$ & & & & \\
\hline Total $(95 \% \mathrm{Cl})$ & & & 561 & 608 & $100.0 \%$ & $0.48[0.25,0.90]$ & & & & \\
\hline \multicolumn{7}{|c|}{$\begin{array}{l}\text { Heterogeneity: } \mathrm{Tau}^{2}=0.27 ; \mathrm{Chi}^{2}=23.62, \mathrm{df}=2(\mathrm{P}<0.00001) ; \mathrm{I}^{2}=92 \% \\
\text { Test for overall effect: } Z=2.30(P=0.02)\end{array}$} & 0.2 & $\begin{array}{c}0.5 \\
\text { Resection }\end{array}$ & ${ }^{1}$ TACE $^{2}$ & 5 \\
\hline
\end{tabular}

Figure 4: The subgroup meta-analysis comparing the overall survival between HCC patients with BCLC stage B alone undergoing hepatic resection and TACE. 
1-year survival rate was statistically similar between the two groups, but 3- and 5-year survival rates were statistically significantly higher in hepatic resection group than in TACE group (Table 2). There were statistically significant heterogeneities in the meta-analyses of 1 - and 3 - year survival rates. The heterogeneity could not be evaluated in the meta-analysis of 5-year survival rate.

\section{Subgroup analysis in patients with PVTT}

In HCC patients with PVTT, the subgroup metaanalysis demonstrated a statistically significantly higher overall survival in hepatic resection group than in TACE group $(\mathrm{HR}=0.78,95 \% \mathrm{CI}=0.68-0.91, \mathrm{P}=0.0009)$ (Figure $5)$. The heterogeneity was statistically significant $(\mathrm{P}=0.06$; $\mathrm{I}^{2}=56 \%$ ). Funnel plots demonstrated that not all studies laid within 95\%CI (Supplementary Figure 5).

Additionally, the meta-analyses demonstrated that 1- and 3-year survival rates were statistically significantly higher in hepatic resection group than in TACE group, but 5-year survival rate was statistically similar between the two groups (Table 2). There was a statistically significant heterogeneity in the meta-analysis of 5- year survival rate, but not in those of 1- and 3-year survival rates.

\section{Sensitivity analyses in moderate- and high-quality studies}

In 16 moderate- and high-quality studies, the sensitivity analysis demonstrated a statistically significantly higher overall survival in hepatic resection group than in TACE group $(\mathrm{HR}=0.62,95 \% \mathrm{CI}=0.53$ $0.71, \mathrm{P}<0.00001$ ) (Figure 6). The heterogeneity was statistically significant $\left(\mathrm{P}<0.00001 ; \mathrm{I}^{2}=83 \%\right)$. Funnel plots demonstrated that not all studies laid within 95\%CI (Supplementary Figure 6).

Additionally, the meta-analyses demonstrated that 1-, 3-, and 5-year survival rates were statistically significantly higher in hepatic resection group than in TACE group (Table 2). There were statistically significant

\begin{tabular}{|c|c|c|c|c|c|c|c|c|c|c|}
\hline Study or Subgroup & log[Hazard Ratio] & SE & $\begin{array}{r}\text { Resection } \\
\text { Total }\end{array}$ & $\begin{array}{l}\text { TACE } \\
\text { Total }\end{array}$ & Weight & $\begin{array}{l}\text { Hazard Ratio } \\
\text { IV. Random. } 95 \% \mathrm{Cl}\end{array}$ & & $\begin{array}{r}\text { Hazard } \\
\text { IV. Randon }\end{array}$ & $\begin{array}{l}\text { d Ratio } \\
\text { m. } 95 \% \mathrm{Cl}\end{array}$ & \\
\hline Cheng 2006 & -0.04 & 0.16 & 7 & 38 & $13.9 \%$ & $0.96[0.70,1.31]$ & & & & \\
\hline Lee JM 2014 & -0.47 & 0.16 & 41 & 80 & $13.9 \%$ & $0.63[0.46,0.86]$ & & & & \\
\hline Liu 2014 & -0.59 & 0.19 & 108 & 108 & $10.9 \%$ & $0.55[0.38,0.80]$ & & & & \\
\hline Peng 2012 & -0.15 & 0.05 & 201 & 402 & $34.0 \%$ & $0.86[0.78,0.95]$ & & + & & \\
\hline Ye 2014 & -0.21 & 0.08 & 90 & 86 & $27.3 \%$ & $0.81[0.69,0.95]$ & & - & & \\
\hline Total $(95 \% \mathrm{Cl})$ & & & 447 & 714 & $100.0 \%$ & $0.78[0.68,0.91]$ & & & & \\
\hline \multicolumn{7}{|c|}{$\begin{array}{l}\text { Heterogeneity: } \mathrm{Tau}^{2}=0.01 ; \mathrm{Chi}^{2}=9.06, \mathrm{df}=4(P=0.06) ; \mathrm{I}^{2}=56 \% \\
\text { Test for overall effect: } Z=3.31(P=0.0009)\end{array}$} & 0.2 & $\begin{array}{ll}0.5 & 1 \\
\text { Resection }\end{array}$ & ${ }^{1}$ TACE $^{2}$ & 5 \\
\hline
\end{tabular}

Figure 5: The subgroup meta-analysis comparing the overall survival between HCC patients with PVTT undergoing hepatic resection and TACE.

\begin{tabular}{|c|c|c|c|c|c|c|c|c|c|c|}
\hline Study or Subgroup & log[Hazard Ratio] & SE & $\begin{array}{l}\text { ection } \\
\text { Total }\end{array}$ & $\begin{array}{l}\text { TACE } \\
\text { Total }\end{array}$ & Weight & $\begin{array}{l}\text { Hazard Ratio } \\
\text { IV. Random. } 95 \% \mathrm{Cl}\end{array}$ & \multicolumn{4}{|c|}{$\begin{array}{c}\text { Hazard Ratio } \\
\text { IV. Random. } 95 \% \mathrm{Cl}\end{array}$} \\
\hline Fan 2014 & -1.32 & 0.3 & 37 & 33 & $3.7 \%$ & $0.27[0.15,0.48]$ & & & & \\
\hline Guo 2014 & -0.39 & 0.09 & 152 & 221 & $7.7 \%$ & $0.68[0.57,0.81]$ & & 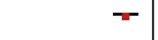 & & \\
\hline Hsu CY 2012 & -0.67 & 0.14 & 146 & 146 & $6.7 \%$ & $0.51[0.39,0.67]$ & & 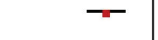 & & \\
\hline Hsu KF 2012 & -0.14 & 0.2 & 112 & 73 & $5.4 \%$ & $0.87[0.59,1.29]$ & & & & \\
\hline Jianyong 2014 & -0.39 & 0.09 & 433 & 490 & $7.7 \%$ & $0.68[0.57,0.81]$ & & $T$ & & \\
\hline Jin 2014 & -0.16 & 0.23 & 62 & 61 & $4.8 \%$ & $0.85[0.54,1.34]$ & & $\longrightarrow$ & & \\
\hline Lin 2010 & -1.24 & 0.15 & 93 & 73 & $6.5 \%$ & $0.29[0.22,0.39]$ & & & & \\
\hline Liu 2014 & -0.59 & 0.19 & 108 & 108 & $5.6 \%$ & $0.55[0.38,0.80]$ & & - & & \\
\hline Luo 2011 & -0.14 & 0.13 & 85 & 83 & $6.9 \%$ & $0.87[0.67,1.12]$ & & & & \\
\hline Min 2014 & -0.7 & 0.17 & 76 & 76 & $6.0 \%$ & $0.50[0.36,0.69]$ & & $=$ & & \\
\hline Peng 2012 & -0.15 & 0.05 & 201 & 402 & $8.4 \%$ & $0.86[0.78,0.95]$ & & - & & \\
\hline Wang 2012 & -0.3 & 0.12 & 119 & 119 & $7.1 \%$ & $0.74[0.59,0.94]$ & & $\rightarrow$ & & \\
\hline Yang 2014 & -1.36 & 0.6 & 52 & 66 & $1.3 \%$ & $0.26[0.08,0.83]$ & & & & \\
\hline Ye 2014 & -0.21 & 0.08 & 90 & 86 & $7.9 \%$ & $0.81[0.69,0.95]$ & & 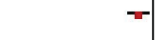 & & \\
\hline Yin 2014 & -0.75 & 0.16 & 88 & 85 & $6.3 \%$ & $0.47[0.35,0.65]$ & & $\neg$ & & \\
\hline Zhong 2014 & -0.43 & 0.08 & 280 & 280 & $7.9 \%$ & $0.65[0.56,0.76]$ & & - & & \\
\hline Total $(95 \% \mathrm{Cl})$ & & & 2134 & 2402 & $100.0 \%$ & $0.62[0.53,0.71]$ & & 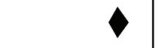 & & \\
\hline \multicolumn{7}{|c|}{$\begin{array}{l}\text { Heterogeneity: } \mathrm{Tau}^{2}=0.07 ; \mathrm{Chi}^{2}=90.83, \mathrm{df}=15(\mathrm{P}<0.00001) ; \mathrm{I}^{2}=83 \% \\
\text { Test for overall effect: } Z=6.40(P<0.00001)\end{array}$} & 0.05 & $\begin{array}{l}0.2 \\
\text { Resection }\end{array}$ & 1 TACE & 20 \\
\hline
\end{tabular}

Figure 6: Sensitivity analysis in moderate- and high-quality studies. 
heterogeneities in all of the 3 meta-analyses.

\section{Sensitivity analyses in studies published after 2005}

In 37 studies published after 2005, the sensitivity analysis demonstrated a statistically significantly higher overall survival in hepatic resection group than in TACE group $(\mathrm{HR}=0.59,95 \% \mathrm{CI}=0.53-0.66, \mathrm{P}<0.00001)$ (Figure 7). The heterogeneity was statistically significant $\left(\mathrm{P}<0.00001 ; \mathrm{I}^{2}=86 \%\right)$. Funnel plots demonstrated that not all studies laid within 95\%CI (Supplementary Figure 7).

Additionally, the meta-analyses demonstrated that 1-, 3-, and 5-year survival rates were statistically significantly higher in hepatic resection group than in
TACE group (Table 2). There were statistically significant heterogeneities in all of the 3 meta-analyses.

\section{DISCUSSION}

In theory, the BCLC staging system needs to be persistently updated with the dramatic improvement in the understanding of $\mathrm{HCC}$ and the invention of novel therapeutic modalities for HCC. Accumulated evidence suggests that the optimal treatment modality of HCC in the BCLC stage B may be further refined. In the present systematic review, we collected the comparative data regarding the overall survival in $\mathrm{HCC}$ patients undergoing hepatic resection and TACE. The overall meta-analysis

\begin{tabular}{|c|c|c|c|c|c|c|c|c|c|c|}
\hline Study or Subqroup & log[Hazard Ratio] & SE & $\begin{array}{l}\text { Resection } \\
\text { Total }\end{array}$ & $\begin{array}{l}\text { TACE } \\
\text { Total }\end{array}$ & Weight & $\begin{array}{l}\text { Hazard Ratio } \\
\text { IV. Random, } 95 \% \mathrm{Cl}\end{array}$ & & $\begin{array}{r}\text { Hazarc } \\
\text { IV. Rando }\end{array}$ & $\begin{array}{l}\text { d Ratio } \\
\text { om, } 95 \% \mathrm{Cl}\end{array}$ & \\
\hline Cheng 2006 & -0.04 & 0.16 & 7 & 38 & $2.7 \%$ & $0.96[0.70,1.31]$ & & & - & \\
\hline Choi 2013 & -0.34 & 0.22 & 36 & 107 & $2.3 \%$ & $0.71[0.46,1.10]$ & & & & \\
\hline Ciria 2014 & -0.58 & 0.33 & 35 & 45 & $1.6 \%$ & $0.56[0.29,1.07]$ & & & & \\
\hline El-Serag 2006 & -0.53 & 0.09 & 243 & 131 & $3.2 \%$ & $0.59[0.49,0.70]$ & & $T$ & & \\
\hline Fan 2014 & -1.32 & 0.3 & 37 & 33 & $1.7 \%$ & $0.27[0.15,0.48]$ & & & & \\
\hline Guglielmi 2011 & -0.72 & 0.11 & 181 & 77 & $3.1 \%$ & $0.49[0.39,0.60]$ & & & & \\
\hline Guo 2014 & -0.39 & 0.09 & 152 & 221 & $3.2 \%$ & $0.68[0.57,0.81]$ & & 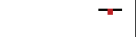 & & \\
\hline Helmberger 2007 & -0.52 & 0.16 & 52 & 107 & $2.7 \%$ & $0.59[0.43,0.81]$ & & 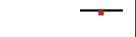 & & \\
\hline Ho 2009 & -0.58 & 0.07 & 294 & 367 & $3.3 \%$ & $0.56[0.49,0.64]$ & & $=$ & & \\
\hline Hsu CY 2012 & -0.67 & 0.14 & 146 & 146 & $2.9 \%$ & $0.51[0.39,0.67]$ & & & & \\
\hline Hsu KF 2012 & -0.14 & 0.2 & 112 & 73 & $2.4 \%$ & $0.87[0.59,1.29]$ & & & & \\
\hline Jianyong 2014 & -0.39 & 0.09 & 433 & 490 & $3.2 \%$ & $0.68[0.57,0.81]$ & & - & & \\
\hline Jin 2014 & -0.16 & 0.23 & 62 & 61 & $2.2 \%$ & $0.85[0.54,1.34]$ & & & & \\
\hline Kang 2010 & -0.7 & 0.39 & 78 & 99 & $1.3 \%$ & $0.50[0.23,1.07]$ & & & & \\
\hline Lee JM 2014 & -0.47 & 0.16 & 41 & 80 & $2.7 \%$ & $0.63[0.46,0.86]$ & & & & \\
\hline Lee YB 2014 & -0.27 & 0.28 & 56 & 56 & $1.9 \%$ & $0.76[0.44,1.32]$ & & & & \\
\hline Lin 2010 & -1.24 & 0.15 & 93 & 73 & $2.8 \%$ & $0.29[0.22,0.39]$ & & & & \\
\hline Liu 2014 & -0.59 & 0.19 & 108 & 108 & $2.5 \%$ & $0.55[0.38,0.80]$ & & & & \\
\hline Luo 2011 & -0.14 & 0.13 & 85 & 83 & $2.9 \%$ & $0.87[0.67,1.12]$ & & & & \\
\hline Min 2014 & -0.7 & 0.17 & 76 & 76 & $2.6 \%$ & $0.50[0.36,0.69]$ & & & & \\
\hline Obed 2008 (1) & -1.14 & 0.75 & 10 & 5 & $0.5 \%$ & $0.32[0.07,1.39]$ & & & & \\
\hline Obed 2008 (2) & -0.42 & 0.27 & 12 & 6 & $1.9 \%$ & $0.66[0.39,1.12]$ & & & & \\
\hline Obed 2008 (3) & 0.09 & 0.09 & 14 & 36 & $3.2 \%$ & $1.09[0.92,1.31]$ & & & 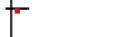 & \\
\hline Park 2008 (1) & -0.48 & 0.26 & 89 & 126 & $2.0 \%$ & $0.62[0.37,1.03]$ & & & & \\
\hline Park 2008 (2) & -0.92 & 0.28 & 28 & 184 & $1.9 \%$ & $0.40[0.23,0.69]$ & & & & \\
\hline Peng 2012 & -0.15 & 0.05 & 201 & 402 & $3.4 \%$ & $0.86[0.78,0.95]$ & & & & \\
\hline Perry 2007 & -0.68 & 0.25 & 43 & 33 & $2.0 \%$ & $0.51[0.31,0.83]$ & & & & \\
\hline Schumacher 2010 & -0.74 & 0.24 & 73 & 47 & $2.1 \%$ & $0.48[0.30,0.76]$ & & & & \\
\hline Sotiropoulos 2009 & -0.13 & 0.18 & 61 & 64 & $2.6 \%$ & $0.88[0.62,1.25]$ & & & & \\
\hline Toro 2014 & -0.63 & 0.21 & 20 & 27 & $2.3 \%$ & $0.53[0.35,0.80]$ & & & & \\
\hline Utsunomiya 2014 & -0.85 & 0.04 & 2872 & 1437 & $3.4 \%$ & $0.43[0.40,0.46]$ & & $=$ & & \\
\hline Wang 2012 & -0.3 & 0.12 & 119 & 119 & $3.0 \%$ & $0.74[0.59,0.94]$ & & & & \\
\hline Wang 2013 & -0.68 & 0.19 & 68 & 140 & $2.5 \%$ & $0.51[0.35,0.74]$ & & & & \\
\hline Worns 2012 & -1.13 & 0.24 & 67 & 23 & $2.1 \%$ & $0.32[0.20,0.52]$ & & & & \\
\hline Yamagiwa 2008 & -0.73 & 0.13 & 101 & 86 & $2.9 \%$ & $0.48[0.37,0.62]$ & & & & \\
\hline Yang 2014 & -1.36 & 0.6 & 52 & 66 & $0.7 \%$ & $0.26[0.08,0.83]$ & & & & \\
\hline Ye 2014 & -0.21 & 0.08 & 90 & 86 & $3.3 \%$ & $0.81[0.69,0.95]$ & & & & \\
\hline Yin 2014 & -0.75 & 0.16 & 88 & 85 & $2.7 \%$ & $0.47[0.35,0.65]$ & & & & \\
\hline Zhang 2014 & -0.65 & 0.12 & 89 & 161 & $3.0 \%$ & $0.52[0.41,0.66]$ & & & & \\
\hline Zhong 2014 & -0.43 & 0.08 & 280 & 280 & $3.3 \%$ & $0.65[0.56,0.76]$ & & 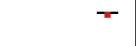 & & \\
\hline Total $(95 \% \mathrm{Cl})$ & & & 6704 & 5884 & $100.0 \%$ & $0.59[0.53,0.66]$ & & $\checkmark$ & & \\
\hline \multicolumn{7}{|c|}{$\begin{array}{l}\text { Heterogeneity: } \mathrm{Tau}^{2}=0.09 ; \mathrm{Chi}^{2}=277.63, \mathrm{df}= \\
\text { Test for overall effect: } \mathrm{Z}=9.44(\mathrm{P}<0.00001)\end{array}$} & 0.05 & $\begin{array}{l}0.2 \\
\text { Resection }\end{array}$ & ${ }^{1}$ TACE & 20 \\
\hline
\end{tabular}

Figure 7: Sensitivity analysis in studies published after 2005. 
demonstrated a statistically significant survival benefit of hepatic resection over TACE. In addition, considering the potential bias of patient selection, we performed several subgroup meta-analyses. All of them confirmed statistically significant survival benefits of hepatic resection over TACE.

At present, the curative treatment options of HCC mainly include liver transplantation (LT), hepatic resection, and radiofrequency ablation (RFA). Although LT is obviously superior to hepatic resection for the complete removal of tumor tissues, it is largely restricted by the scarcity of liver donors. On the other hand, hepatic resection appears to be superior to RFA for the improvement of overall survival and recurrencefree survival in HCC within the Milan criteria [56]. In clinical practices, hepatic resection is often regarded as the primary choice of therapy for early stage HCC, if the lesion is single, hepatic function is well-preserved, and portal hypertension is not severe. Under this circumstance, the results of our subgroup meta-analysis that hepatic resection could achieve a significantly better survival than TACE in HCC within the BCLC stage A were in line with our expectations.

The non-curative treatment options of HCC mainly include TACE and sorafenib. TACE is the firstline treatment option of $\mathrm{HCC}$ in the BCLC stage B. This recommendation is primarily attributed to the survival benefits of TACE over conservative or suboptimal treatments [57]. But we are not sure about whether TACE surpasses other active treatments for the improvement of overall survival. Our subgroup meta-analyses suggested that the survival was statistically significantly better in HCC patients beyond the BCLC stage A undergoing hepatic resection than in those undergoing TACE. Thus, hepatic resection might be also considered in selected HCC cases in the BCLC stage B.

After our study was registered, Kapitanov and colleagues published a similar meta-analysis to compare the short- and long-term results of hepatic resection versus TACE in HCC patients with cirrhosis [58]. They also concluded that the survival at 1 and 3 years was significantly better in patients treated with surgery than in those treated with TACE. Compared with their study, our systematic review and meta-analysis had several strengths. First, the selection of target population was broader and the number of included studies was larger. Thus, we were permitted to conduct more subgroup meta-analyses according to the study and patient characteristics. Second, the study quality was strictly assessed. Thus, we could readily understand the grade of current evidence. Third, the duplicate data were excluded, thereby avoiding the inflation of relevant information [59]. In the meta-analysis by Kapitanov et al., two papers by Zhong et al. were included. However, they reported the overlapping data. In the first paper, $392 \mathrm{HCC}$ patients in the BCLC stage
B were enrolled between January 2000 and November 2007 [60]; and in the second paper, $860 \mathrm{HCC}$ patients in the BCLC stages B and C were enrolled between January 2000 and November 2007 [55]. By comparison, the first paper with a smaller sample size was excluded from our meta-analysis. Indeed, four other papers conducted by the same study team were also excluded from our metaanalysis [61-64].

The limitations of our meta-analysis should be clearly emphasized. First, only one included study was a randomized controlled trial. Additionally, a majority of included studies were of low quality. Certainly, we conducted a subgroup meta-analysis of moderate- and high-quality studies to confirm the reliability of our findings. Second, the heterogeneity among studies was statistically significant in all but one meta-analysis of HCC cases within the BCLC stage A. We employed a random-effect model to produce a relatively conservative estimate. Third, the publication bias was statistically significant in a majority of meta-analyses, despite three major English-language databases were searched. Fourth, the overall survival was the sole outcome observed in our study. Thus, we could not capture the other advantages or disadvantages of hepatic resection versus TACE. However, it should be noted that the overall survival was the most important endpoint to measure the therapeutic effectiveness in HCC [65]. By contrast, the time to recurrence, progression-free survival, and disease-free survival were the secondary endpoints. We could hardly translate the improvements in these secondary endpoints into the clinical practice recommendations.

In conclusions, hepatic resection might provide a better overall survival than TACE in HCC beyond the BCLC stage A. However, we should acknowledge that the current evidence is of low-quality. Considering that the drawbacks of study designs potentially lead to the selection biases, more well-designed randomized controlled trials should be warranted to compare the survival benefit of hepatic resection versus TACE in such patients.

\section{METERIALS AND METHODS}

This work was registered with PROSPERO on December 19, 2014 (registration number: CRD42014015618).

\section{Search strategy}

The PubMed, EMBASE, and Cochrane Library databases were searched. Search items were as follows: ("Hepatectomy" OR "Liver resection" OR "Hepatic resection" OR "Liver surgery" OR "Hepatic surgery") AND ("TACE" OR "transarterial chemoembolization") 
AND ("HCC" OR "hepatocellular carcinoma" OR "hepatic carcinoma"). The last search was performed on December 18, 2014. Relevant literatures were also manually searched.

\section{Study selection}

Only clinical studies including more than 10 patients were considered in the systematic review. Accordingly, duplicate papers among databases, redundant publications, narrative or systematic reviews, study protocols, comments, experimental studies, and case reports were excluded. If two or more papers by the same study team had the overlapping data, only one paper with more adequate data and/or a longer enrollment period would be included.

The inclusion criteria should be as follows.

Participants: patients with HCC.

Interventions: hepatic resection and TACE as initial treatment modalities.

Comparisons: hepatic resection versus TACE.

Outcomes: overall survival.

The exclusion criteria should be as follows.

1) Non-HCC.

2) Hepatic metastases.

3) Mixed malignancies.

4) Non-comparative studies. TACE.

5) No comparison between hepatic resection versus

6) TACE before and after hepatic resection.

7) Comparison between hepatic resection versus TACE for recurrent HCC.

8) Comparison between hepatic resection versus TACE for spontaneous rupture of HCC.

9)No separate data in the hepatic resection or TACE group.

10) No detailed data regarding the survival rate in the hepatic resection or TACE group.

11) No detailed data regarding the number of observed patients in the hepatic resection or TACE group.

Notably, the major reason for exclusion of studies including patients with recurrent $\mathrm{HCC}$ and spontaneous rupture of $\mathrm{HCC}$ was the discrepancy in the treatment selection and outcomes among them.

\section{Data extraction}

The following data were extracted: the first author, publication year, publication form, region, enrollment period, study design, study population, follow-up time, inclusion and exclusion criteria, number of HCC cases, treatment selection, survival rate, survival times, and Kaplan-Meier curve analysis with log-rank test. If the propensity score matching analysis was performed, we just collected the survival data after the propensity score matching analyses. If both survival rates and KaplanMeier curves were presented, only the survival rates would be collected. If only Kaplan-Meier curves were presented, we extracted the cumulative 1-, 3-, and 5-year survival rates by using the Distance Tool in the Measurements menu of Foxit PDF Reader software version 5.4.4.1023 (Foxit Cooperation, California, USA). This software was freely downloaded.

\section{Study quality}

Because both retrospective/prospective observational studies and randomized controlled trials were included in the present systematic review, we could not employ a single scale to evaluate the quality of all included studies. More importantly, because our study was designed to compare the overall survival between patients undergoing hepatic resection and those undergoing TACE, the study quality assessment should primarily focus on the comparability of patient characteristics between the two groups. According to the Newcastle-Ottawa scale and major prognostic factors of HCC [66], we developed the following 9 questions that were more specific to the study quality assessment in the present systematic review.

1) Were the patients consecutively enrolled and prospectively followed?

2) Was the age statistically similar between the two groups?

3) Was the gender statistically similar between the two groups?

4) Was the Child-Pugh score/class or MELD score statistically similar between the two groups?

5) Were the diameter and number of tumor statistically similar between the two groups?

6) Was the BCLC stage or other HCC stage statistically similar between the two groups?

7) Were the criteria for treatment selection homogeneous between the two groups?

8) Was the follow-up time clearly reported?

9) Was the proportion of patients lost to follow-up less than $20 \%$ ?

If the answers to 7-9 questions were "Yes", the study would be considered to be of high quality. If the answers to 4-6 questions were "Yes", the study would be considered to be of moderate quality. Otherwise, it would be considered to be of poor quality.

\section{Meta analysis}

Only a minority of included studies clearly reported the hazard ratios (HRs) for the overall survival in HCC patients with hepatic resection versus TACE. Therefore, we calculated $\ln [\mathrm{HR}]$ with standard error by using the 
calculation sheets which were developed by Matthew Sydes and Jayne Tierney [67]. The survival rates at different time points were entered into the calculation sheet of "(2a) curve data". Accordingly, a curve was produced in the calculation sheet of " $(2 \mathrm{~b})$ curve copy", and $\ln [\mathrm{HR}]$ and $\operatorname{se}(\ln [\mathrm{HR}])$ could be available in the calculation sheet of “(4) output information”. Then, HRs with 95\% confidence intervals (CIs) were pooled by using a random-effects model. Additionally, to provide the survival data in detail, we compared the 1-, 3-, and 5 -year survival rates between HCC patients with hepatic resection versus TACE. The odd ratios (ORs) with 95\% CIs were pooled by using a random random-effects model. In these meta-analyses, a $\mathrm{P}$ value of $<0.05$ was considered statistically significant. Heterogeneity between studies was assessed by using the $\mathrm{I}^{2}$ statistic $\left(\mathrm{I}^{2}>50 \%\right.$ was considered as having substantial heterogeneity) and the Chi-square test $(\mathrm{P}<0.10$ was considered to represent significant statistical heterogeneity). Funnel plots were performed to evaluate the publication bias. Subgroup meta-analyses were performed according to the BCLC stages (within versus beyond BCLC stage A). Subgroup difference between the two groups was evaluated by using the $\mathrm{I}^{2}$ statistic $\left(\mathrm{I}^{2}>50 \%\right.$ was considered as having statistically significant difference) and the Chi-square test $(\mathrm{P}<0.10$ was considered to represent statistically significant difference). Subgroup meta-analyses were also performed in patients with BCLC stage B alone and in those with portal vein tumor thrombus (PVTT). Sensitivity analyses were performed in moderate- and high-quality studies and studies published after 2005. All metaanalyses were conducted by using the statistical package Review Manager version 5.1.6 (Copenhagen, The Nordic Cochrane Center, The Cochrane Collaboration, 2011).

\section{Author contributions}

XQ: designed the study, performed the literature search and selection, data extraction, quality assessment, and statistical analysis, and drafted the manuscript; DW: performed the literature selection, data extraction, and quality assessment; CS: performed the literature search; HL and XG: gave critical comments and revised the manuscript. All authors have made an intellectual contribution to the manuscript and approved the submission.

\section{ACKNOWLEDGMENTS AND FUNDING}

None.

\section{CONFLICTS OF INTEREST}

The authors report no declarations of interest.

\section{REFERENCES}

1. Bruix $\mathbf{J}$ and Sherman M. Management of hepatocellular carcinoma: an update. Hepatology. 2011; 53:1020-1022.

2. EASL-EORTC clinical practice guidelines: management of hepatocellular carcinoma. J Hepatol. 2012; 56:908-943.

3. Farinati F, Vanin V, Giacomin A, Pozzan C, Cillo U, Vitale A, Di Nolfo AM, Del Poggio P, Benvegnu L, Rapaccini G, Zoli M, Borzio F, Giannini EG, Caturelli E and Trevisani F. BCLC stage B hepatocellular carcinoma and transcatheter arterial chemoembolization: a 20-year survey by the Italian Liver Cancer group. Liver Int. 2015; 35:223-231.

4. Vitale A, Burra P, Frigo AC, Trevisani F, Farinati F, Spolverato G, Volk M, Giannini EG, Ciccarese F, Piscaglia F, Rapaccini GL, Di Marco M, Caturelli E, Zoli M, Borzio F, Cabibbo G, et al. Survival benefit of liver resection for patients with hepatocellular carcinoma across different Barcelona Clinic Liver Cancer stages: A multicentre study. J Hepatol. 2015; 62:617-624.

5. Roayaie S, Jibara G, Tabrizian P, Park JW, Yang J, Yan L, Schwartz M, Han G, Izzo F, Chen M, Blanc JF, Johnson P, Kudo M, Roberts LR and Sherman M. The Role of hepatic resection in the treatment of hepatocellular cancer. Hepatology. 2015; 62:440-51.

6. Cheng SQ, Wu MC, Chen H, Shen F, Yang JH, Cong WM, Zhao YX, Wang PJ and Ding GH. Hepatocellular carcinoma with tumor thrombi in the portal vein. A comparison of therapeutic effects by different treatments. Zhonghua zhong liu za zhi [Chinese journal of oncology]. 2005; 27:183-185.

7. Choi SH, Choi GH, Kim SU, Park JY, Joo DJ, Ju MK, Kim MS, Choi JS, Han KH and Kim SI. Role of surgical resection for multiple hepatocellular carcinomas. World J Gastroenterol. 2013; 19:366-374.

8. Ciria R, Gallardo AB, Cabrera J, Luque A, Ayllon MD, Rodriguez-Peralvarez M, Montero JL, Lopez-Cillero P, De La Mata $\mathrm{M}$ and Briceno J. Modern surgical liver resections offer better results than transarterial chemoembolization in cirrhotic patients with B-BCLC stage hepatocellular carcinoma. Journal of Hepatology. 2014; 60:S402.

9. Colella G, Bottelli R, De Carlis L, Sansalone CV, Rondinara GF, Alberti A, Belli LS, Gelosa F, Iamoni GM, Rampoldi A, De Gasperi A, Corti A, Mazza E, Aseni P, Meroni A, Slim AO, et al. Hepatocellular carcinoma: comparison between liver transplantation, resective surgery, ethanol injection, and chemoembolization. Transplant international : official journal of the European Society for Organ Transplantation. 1998; 11 Suppl 1:S193-196.

10. El-Serag HB, Siegel AB, Davila JA, Shaib YH, CaytonWoody M, McBride R and McGlynn KA. Treatment and outcomes of treating of hepatocellular carcinoma among Medicare recipients in the United States: a population-based study. J Hepatol. 2006; 44:158-166.

11. Fan HL, Hsieh CB, Chang WC, Huang SH, Chan DC, 
$\mathrm{Yu}$ JC, Chu $\mathrm{CH}$ and Chen TW. Advanced age is not a contraindication for liver resection in cases of large hepatocellular carcinoma. Eur J Surg Oncol. 2014; 40:214219.

12. Gerunda GE, Neri D, Merenda R, Barbazza F, Zangrandi F, Meduri F, Bisello M, Valmasoni M, Gangemi A and Faccioli AM. Role of transarterial chemoembolization before liver resection for hepatocarcinoma. Liver Transpl. 2000; 6:619-626.

13. Guglielmi A, Ruzzenente A, Capra F, Campagnaro T, Valdegamberi A, Ieluzzi D, Turcato $G$ and Iacono C. Surgical versus non surgical therapy of hec on cirrhosis: Results of an observational study on 692 cases. HPB. 2011; $13: 40$.

14. Guo Z, Zhong JH, Jiang JH, Zhang J, Xiang BD and Li LQ. Comparison of survival of patients with BCLC stage A hepatocellular carcinoma after hepatic resection or transarterial chemoembolization: a propensity score-based analysis. Ann Surg Oncol. 2014; 21:3069-3076.

15. Hasse F, Mehring UM, Castaneda F, Jager H, Mathias $\mathrm{KD}$ and Lohlein D. Comparison of liver resection with sequential transarterial chemoembolization in stage pT3 or pT4 hepatocellular carcinoma. Langenbecks Archiv für Chirurgie Supplement Kongressband Deutsche Gesellschaft für Chirurgie Kongress. 1996; 113:208-210.

16. Helmberger T, Dogan S, Straub G, Schrader A, Jungst C, Reiser M, Waggershauser T, Jakobs T, Hoffmann RT, Lohe F, Graeb C, Rau HG, Schauer R, Jauch KW, Caselmann WH, Goke B, et al. Liver resection or combined chemoembolization and radiofrequency ablation improve survival in patients with hepatocellular carcinoma. Digestion. 2007; 75:104-112.

17. Herold C, Reck T, Fischler P, Ott R, Radespiel-Troeger M, Ganslmayer M, Hohenberger W, Hahn EG and Schuppan D. Prognosis of a large cohort of patients with hepatocellular carcinoma in a single European centre. Liver. 2002; 22:2328.

18. Ho MC, Huang GT, Tsang YM, Lee PH, Chen DS, Sheu $\mathrm{JC}$ and Chen $\mathrm{CH}$. Liver resection improves the survival of patients with multiple hepatocellular carcinomas. Ann Surg Oncol. 2009; 16:848-855.

19. Hsu CY, Hsia CY, Huang YH, Su CW, Lin HC, Pai JT, Loong CC, Chiou YY, Lee RC, Lee FY, Huo TI and Lee $\mathrm{SD}$. Comparison of surgical resection and transarterial chemoembolization for hepatocellular carcinoma beyond the Milan criteria: a propensity score analysis. Ann Surg Oncol. 2012; 19:842-849.

20. Hsu KF, Chu CH, Chan DC, Yu JC, Shih ML, Hsieh HF, Hsieh TY, Yu CY and Hsieh CB. Superselective transarterial chemoembolization vs hepatic resection for resectable early-stage hepatocellular carcinoma in patients with Child-Pugh class a liver function. Eur J Radiol. 2012; 81:466-471.

21. Huang YH, Wu JC, Chau GY, Lui WY, King KL, Chiang JH, Yen SH, Sheng WY, Hou MC, Lu CL, Chang FY and
Lee SD. Supportive treatment, resection and transcatheter arterial chemoembolization in resectable hepatocellular carcinoma: an analysis of survival in 419 patients. Eur J Gastroenterol Hepatol. 1999; 11:315-321.

22. Jianyong L, Lunan Y, Wentao W, Yong Z, Bo L, Tianfu $\mathrm{W}$, Minqing $\mathrm{X}$ and Jiaying $\mathrm{Y}$. Barcelona clinic liver cancer stage B hepatocellular carcinoma: transarterial chemoembolization or hepatic resection? Medicine (Baltimore). 2014; 93:e180.

23. Jin YJ, Lee JW, Choi YJ, Chung HJ, Kim YS, Lee KY, Ahn SI, Shin WY, Cho SG and Jeon YS. Surgery versus transarterial chemoembolization for solitary large hepatocellular carcinoma of BCLC stage A. J Gastrointest Surg. 2014; 18:555-561.

24. Kang S, Kim SU, Choi GH, Choi JS, Park JY, Lee KH, Lee DY, Kim DY, Ahn SH, Han KH and Chon CY. Comparing the outcomes of transarterial chemoembolization and hepatic resection in patients with single hepatocellular carcinoma less than $3 \mathrm{~cm}$. Hepatology International. 2010; 4:304-305.

25. Kirchner G, Hebestreit A, Farkas S, Zorger N, Schnitzbauer A, Hartl J, Loss M, Baier L, Wiest R, Scherer MN and Schlitt HJ. Less than $10 \%$ of patients with hepatocellular carcinoma qualify for liver transplantation and show best long-term survival rates. Transplant International. 2011; $24: 20$.

26. Lee JM, Jang BK, Chung WJ, Hwang JS, Kang KJ, Kim YH, Kweon YO, Park SY, Tak WY, Kim BS and Lee CH. The survival outcomes of hepatic resection compared with transcatheter arterial chemoembolization for hepatocellular carcinoma with portal vein tumor thrombus. Hepatology International. 2014; 8:S22-S23.

27. Lee YB, Lee DH, Cho Y, Yu SJ, Lee JH, Yoon JH, Lee HS, Kim HC, Yi NJ, Lee KW, Suh KS, Chung JW and Kim YJ. Comparison of chemoembolization and hepatic resection for large solitary hepatocellular carcinoma: A propensity score analysis. Journal of Hepatology. 2014; 60:S398.

28. Lin CT, Hsu KF, Chen TW, Yu JC, Chan DC, Yu CY, Hsieh TY, Fan HL, Kuo SM, Chung KP and Hsieh CB. Comparing hepatic resection and transarterial chemoembolization for Barcelona Clinic Liver Cancer (BCLC) stage B hepatocellular carcinoma: change for treatment of choice? World J Surg. 2010; 34:2155-2161.

29. Liu PH, Lee YH, Hsia CY, Hsu CY, Huang YH, Chiou YY, Lin HC and Huo TI. Surgical resection versus transarterial chemoembolization for hepatocellular carcinoma with portal vein tumor thrombosis: a propensity score analysis. Ann Surg Oncol. 2014; 21:1825-1833.

30. Luo J, Peng ZW, Guo RP, Zhang YQ, Li JQ, Chen MS and Shi M. Hepatic resection versus transarterial lipiodol chemoembolization as the initial treatment for large, multiple, and resectable hepatocellular carcinomas: a prospective nonrandomized analysis. Radiology. 2011; 259:286-295.

31. Markovic S, Gadzijev E, Stabuc B, Croce LS, Masutti F, 
Surlan M, Berden P, Brencic E, Visnar-Perovic A, Sasso F, Ferlan-Marolt V, Mucelli FP, Cesar R, Sponza M and Tiribelli C. Treatment options in Western hepatocellular carcinoma: a prospective study of 224 patients. J Hepatol. 1998; 29:650-659.

32. Martins A, Cortez-Pinto H, Marques-Vidal P, Mendes N, Silva S, Fatela N, Gloria H, Marinho R, Tavora I, Ramalho $\mathrm{F}$ and de Moura MC. Treatment and prognostic factors in patients with hepatocellular carcinoma. Liver Int. 2006; 26:680-687.

33. Min YW, Lee JH, Gwak GY, Paik YH, Rhee PL, Koh KC, Paik SW, Yoo BC and Choi MS. Long-term survival after surgical resection for huge hepatocellular carcinoma: comparison with transarterial chemoembolization after propensity score matching. J Gastroenterol Hepatol. 2014; 29:1043-1048.

34. Nagashima J, Okuda K, Tanaka M, Sata M and Aoyagi S. Prognostic benefit in cytoreductive surgery for curatively unresectable hepatocellular carcinoma - comparison to transcatheter arterial chemoembolization. Int J Oncol. 1999; 15:1117-1123.

35. Obed A, Tsui TY, Schnitzbauer AA, Obed M, Schlitt HJ, Becker $\mathrm{H}$ and Lorf $\mathrm{T}$. Liver transplantation as curative approach for advanced hepatocellular carcinoma: is it justified? Langenbecks Arch Surg. 2008; 393:141-147.

36. Park KW, Park JW, Choi JI, Kim TH, Kim SH, Park HS, Lee WJ, Park SJ, Hong EK and Kim CM. Survival analysis of 904 patients with hepatocellular carcinoma in a hepatitis B virus-endemic area. J Gastroenterol Hepatol. 2008; 23:467-473.

37. Paul SB, Chalamalasetty SB, Vishnubhatla S, Madan K, Gamanagatti SR, Batra Y, Gupta SD, Panda SK and Acharya SK. Clinical profile, etiology and therapeutic outcome in 324 hepatocellular carcinoma patients at a tertiary care center in India. Oncology. 2009; 77:162-171.

38. Peng ZW, Guo RP, Zhang YJ, Lin XJ, Chen MS and Lau WY. Hepatic resection versus transcatheter arterial chemoembolization for the treatment of hepatocellular carcinoma with portal vein tumor thrombus. Cancer. 2012; 118:4725-4736.

39. Perry JF, Charlton B, Koorey DJ, Waugh RC, Gallagher PJ, Crawford MD, Verran DJ, McCaughan GW and Strasser SI. Outcome of patients with hepatocellular carcinoma referred to a tertiary centre with availability of multiple treatment options including cadaveric liver transplantation. Liver Int. 2007; 27:1240-1248.

40. Sako K, Komorizono Y, Oketani M, Hasegawa S, Yamasaki N, Shibatou T, Maeda M, Kohara K, Shigenobu S, Yasunari H, Sakurai K, Hiwaki T, Ishibashi K and Arima T. Outcome of Patients with Hepatitis C Virus-related Single, Small Hepatocellular Carcinoma. Anticancer Research. 2003; 23:4191-4196.

41. Sasaki Y, Imaoka S, Nakano H, Ishikawa O, Ohigashi H, Yasuda T, Nakamori S, Kameyama M, Hiratsuka M, Kabuto T, Furukawa H, Kasugai H, Inoue A, Fujita M and
Inoue E. Indications for hepatectomy for hepatocellular carcinoma - what stage of the disease is the best indication for surgery? J Hepatobiliary Pancreat Surg. 1998; 5:14-17.

42. Schumacher PA, Powell JJ, MacNeill AJ, Buczkowski AK, Erb SR, Ho SG, Scudamore CH, Steinbrecher UP, Weiss A, Yoshida E and Chung SW. Multimodal therapy for hepatocellular carcinoma: a complementary approach to liver transplantation. Ann Hepatol. 2010; 9:23-32.

43. Sotiropoulos GC, Druhe N, Sgourakis G, Molmenti EP, Beckebaum S, Baba HA, Antoch G, Hilgard P, Radtke A, Saner FH, Nadalin S, Paul A, Malago M, Broelsch $\mathrm{CE}$ and Lang $\mathrm{H}$. Liver transplantation, liver resection, and transarterial chemoembolization for hepatocellular carcinoma in cirrhosis: which is the best oncological approach? Dig Dis Sci. 2009; 54:2264-2273.

44. Toro A, Ardiri A, Mannino M, Arcerito MC, Mannino G, Palermo F, Bertino G and Di Carlo I. Effect of pre- and post-treatment alpha-fetoprotein levels and tumor size on survival of patients with hepatocellular carcinoma treated by resection, transarterial chemoembolization or radiofrequency ablation: a retrospective study. BMC Surg. 2014; 14:40.

45. Ueno S, Tanabe G, Nuruki K, Oketani M, Komorizono Y, Hokotate H, Fukukura Y, Baba Y, Imamura Y and Aikou T. Prognosis of hepatocellular carcinoma associated with Child class B and C cirrhosis in relation to treatment: a multivariate analysis of 411 patients at a single center. J Hepatobiliary Pancreat Surg. 2002; 9:469-477.

46. Utsunomiya T, Shimada M, Kudo M, Ichida T, Matsui O, Izumi N, Matsuyama Y, Sakamoto M, Nakashima $\mathrm{O}, \mathrm{Ku}$ Y, Kokudo N and Makuuchi M. Nationwide study of 4741 patients with non-B non-C hepatocellular carcinoma with special reference to the therapeutic impact. Ann Surg. 2014; 259:336-345.

47. Wang Sz, Meng Wj, An HQ, Shi Fs and Wang Xl. Outcome evaluation of hepatocellular carcinoma patients after balancing the covariates between groups through propensity score. Academic Journal of Second Military Medical University. 2012; 33:1090-1094.

48. Wang JH, Kuo YH, Wang CC, Chen CL, Cheng YF, Hsu $\mathrm{HC}$ and Lu SN. Surgical resection improves the survival of selected hepatocellular carcinoma patients in Barcelona clinic liver cancer stage C. Dig Liver Dis. 2013; 45:510515.

49. Worns MA, Bosslet T, Victor A, Koch S, HoppeLotichius M, Heise M, Hansen T, Pitton MB, Niederle IM, Schuchmann M, Weinmann A, Duber C, Galle PR and Otto G. Prognostic factors and outcomes of patients with hepatocellular carcinoma in non-cirrhotic liver. Scand J Gastroenterol. 2012; 47:718-728.

50. Yamagiwa K, Shiraki K, Yamakado K, Mizuno S, Hori T, Yagi S, Hamada T, Iida T, Nakamura I, Fujii K, Usui $\mathrm{M}$, Isaji S, Ito K, Tagawa S, Takeda K, Yokoi H, et al. Survival rates according to the Cancer of the Liver Italian Program scores of 345 hepatocellular carcinoma patients 
after multimodality treatments during a 10-year period in a retrospective study. J Gastroenterol Hepatol. 2008; 23:482490.

51. Yang HJ, Lee JH, Lee DH, Yu SJ, Kim YJ, Yoon JH, Kim HC, Lee JM, Chung JW, Yi NJ, Lee KW, Suh KS and Lee HS. Small single-nodule hepatocellular carcinoma: comparison of transarterial chemoembolization, radiofrequency ablation, and hepatic resection by using inverse probability weighting. Radiology. 2014; 271:909918.

52. Ye JZ, Zhang YQ, Ye HH, Bai T, Ma L, Xiang BD and Li LQ. Appropriate treatment strategies improve survival of hepatocellular carcinoma patients with portal vein tumor thrombus. World J Gastroenterol. 2014; 20:17141-17147.

53. Yin L, Li H, Li AJ, Lau WY, Pan ZY, Lai EC, Wu MC and Zhou WP. Partial hepatectomy vs. transcatheter arterial chemoembolization for resectable multiple hepatocellular carcinoma beyond Milan Criteria: a RCT. J Hepatol. 2014; 61:82-88.

54. Zhang T, Zeng Y, Huang J, Liao M and Wu H. Combined resection with radiofrequency ablation for bilobar hepatocellular carcinoma: a single-center experience. J Surg Res. 2014; 191:370-378.

55. Zhong JH, Ke Y, Gong WF, Xiang BD, Ma L, Ye XP, Peng T, Xie GS and Li LQ. Hepatic resection associated with good survival for selected patients with intermediate and advanced-stage hepatocellular carcinoma. Ann Surg. 2014; 260:329-340.

56. Qi X, Tang Y, An D, Bai M, Shi X, Wang J, Han G and Fan D. Radiofrequency ablation versus hepatic resection for small hepatocellular carcinoma: a meta-analysis of randomized controlled trials. J Clin Gastroenterol. 2014; 48:450-457.

57. Llovet JM and Bruix J. Systematic review of randomized trials for unresectable hepatocellular carcinoma: Chemoembolization improves survival. Hepatology. 2003; 37:429-442.

58. Kapitanov T, Neumann UP and Schmeding $M$. Hepatocellular Carcinoma in Liver Cirrhosis: Surgical Resection versus Transarterial Chemoembolization-A MetaAnalysis. Gastroenterol Res Pract. 2015; 2015:696120.

59. Qi X, Yang M, Ren W, Jia J, Wang J, Han G and Fan D. Find duplicates among the PubMed, EMBASE, and Cochrane Library Databases in systematic review. PLoS One. 2013; 8:e71838.

60. Zhong JH, Xiang BD, Gong WF, Ke Y, Mo QG, Ma L, Liu $\mathrm{X}$ and Li LQ. Comparison of long-term survival of patients with BCLC stage B hepatocellular carcinoma after liver resection or transarterial chemoembolization. PLoS One. 2013; 8:e68193.

61. Guo Z, Xiang B, Zhang J, Jiang J, Li L. Comparison of hepatic resection and transarterial chemoembolization for hepatocellular carcinoma within the Milan criteria [Article in Chinese]. Zhonghua Yi Xue Za Zhi. 2014; 94:1526-9.
62. Guo Z, Zhong JH, Jiang JH, Zhang J, Xiang BD and Li LQ. Comparison of survival of patients with BCLC stage a hepatocellular carcinoma after hepatic resection or transarterial chemoembolization: A propensity score-based analysis. Annals of Surgical Oncology. 2014; 21:30693076.

63. Ke Y, Zhong J, Guo Z, Liang Y, Li L, Xiang B. Comparison liver resection with transarterial chemoembolization for Barcelona Clinic Liver Cancerstage B hepatocellular carcinoma patients on long-term survival after SPSS propensity scorematching [Article in Chinese]. Zhonghua Yi Xue Za Zhi. 2014; 94:747-50.

64. Liu X, Li L, Xiang B, Ma L, Zhong J and You X. Efficacy of hepatic resection and transarterial chemoembolization for stage B hepatocellular carcinoma in the Barcelona Clinic Liver Cancer classification. Chinese Journal of Clinical Oncology. 2012; 39:1225-1228.

65. Llovet JM, Di Bisceglie AM, Bruix J, Kramer BS, Lencioni R, Zhu AX, Sherman M, Schwartz M, Lotze M, Talwalkar $\mathrm{J}$ and Gores GJ. Design and endpoints of clinical trials in hepatocellular carcinoma. J Natl Cancer Inst. 2008; 100:698-711.

66. Wells GA, Shea B, O'Connell D, Peterson J, Welch V, Losos $\mathrm{M}$ and Tugwell P. The Newcastle-Ottawa Scale (NOS) for assessing the quality of nonrandomised studies in meta-analyses. http://wwwohrica/programs/clinical_ epidemiology/oxfordasp.

67. Tierney JF, Stewart LA, Ghersi D, Burdett S and Sydes MR. Practical methods for incorporating summary time-toevent data into meta-analysis. Trials. 2007; 8:16. 